\title{
FinIrrSDA: A 3D model for magnetic shape and distribution anisotropy of finite irregular arrangements of particles with different sizes, geometries, and orientations
}

Andrea Regina Biedermann

Institute of Geological Sciences, University of Bern, Baltzerstrasse 1+3, 3012 Bern, Switzerland andrea.regina.biedermann@gmail.com

\section{Key points}

Model to predict magnetic shape and distribution anisotropy for finite assemblies of particles with unequal size, shape and orientation

More accurate representation of grains or pores in natural samples than existing code improves fit with experimental data

May help solve controversy on importance of shape vs distribution anisotropy, and facilitate interpretation of magnetic (pore) fabrics

The final version of the article is available at:

https://agupubs.onlinelibrary.wiley.com/doi/abs/10.1029/2020JB020300 


\section{Abstract}

The magnetic anisotropy carried by strongly magnetic particles such as magnetite or ferrofluid-filled pores is generally composed of shape anisotropy and distribution anisotropy. Their relative importance in rocks depends on numerous factors and has been discussed controversially. A major challenge in estimating their contributions so far has been that models for distribution anisotropy only exist for regular arrangements of equal particles along lines or in planes. Because magnetite grains or pores in rocks display wide ranges of orientations, shapes and sizes in generally irregular arrangements, new models are needed to describe distribution anisotropy for more realistic grain and pore assemblies. The model presented in this study, FinIrrSDA, calculates Shape and Distribution Anisotropy for FINite IRRegular assemblies of unequal particles with different orientations. Input parameters are provided as a table with $x, y$ and $z$ coordinates of the particle centers, and the lengths and orientations of the major, intermediate and minor axes of best-fit ellipsoids. The model output consists of two susceptibility tensors: (1) the shape anisotropy tensor, and (2) the total tensor combining shape and distribution anisotropies. FinIrrSDA can be applied to a wide range of input datasets, including known structures of synthetic samples, particle analyses from tomography data, and, subject to certain assumptions, 2D images. The model will hopefully increase our understanding of the interplay between shape and distribution anisotropies in natural rocks, and facilitate future interpretations of both the magnetic anisotropy carried by magnetite grains, and magnetic pore fabrics.

\section{Plain language summary}

Models are helpful when we aim at understanding and interpreting measured data, provided that they are applicable to reality. This paper presents a new model to predict magnetic properties and their directional dependence (anisotropy) of strongly magnetic particles (e.g. magnetite or pores filled with strongly magnetic fluid) in rock samples. This model is applicable to irregular assemblies of particles of various sizes and orientations, and thus helps close the gap between existing models and reality. The model helps us understand the sources of magnetic anisotropy, and thus makes magnetic anisotropy an even more valuable tool to investigate preferred alignment of magnetite or pores in rocks.

\section{Introduction}

Magnetic fabrics are commonly used in tectonic and geodynamic studies to characterize mineral alignment, which in turn reflects deformation processes and geologic history (Borradaile \& Henry, 1997; Borradaile \& Jackson, 2004; Borradaile \& Jackson, 2010; Hrouda, 1982; Jackson \& Tauxe, 1991; Jackson, 1991; Tarling \& Hrouda, 1993). Additionally, magnetic anisotropy measured on samples impregnated with strongly magnetic fluid (ferrofluid) provides information on the pore fabric, which can be related to permeability anisotropy and preferred flow directions (Hailwood et al., 1999; Pares et al., 2016; Pfleiderer \& Halls, 1990, 1994). In a first approximation, magnetic anisotropy may be interpreted based on empirical relationships. Commonly used empirical relationships to relate magnetic anisotropy to mineral alignment are that (1) the maximum and minimum principal susceptibilities indicate the macroscopic lineation and pole of the foliation plane, as observed in granitic rocks (Balsley \& Buddington, 1960), and (2) the degree of anisotropy is linked to the degree of deformation (Hirt et al., 1988; Kligfield et al., 1977; Kligfield et al., 1981). While empirical relationships can be powerful proxies, they may be largely affected by mineralogy (Borradaile, 1987; Housen et al., 1993). Thus, more robust and quantitative interpretations require a detailed and thorough understanding of the physical origin(s) and carrier minerals of the magnetic anisotropy. This is especially important when fabrics are complex superpositions of contributions of various 
minerals that may have formed at different times during a rock's history, in which case empirical relationships may not hold (Biedermann et al., 2018; Martín-Hernández \& Ferré, 2007; Rochette et al., 1992). Analogously, empirical relationships used to interpret pore fabrics state that (1) the maximum principal susceptibility of a ferrofluid-impregnated sample indicates the direction of pore elongation and maximum permeability, and (2) the degree of anisotropy correlates with the pore shape and the permeability anisotropy (Pfleiderer \& Halls, 1990,1994). However, the details of these empirical relationships vary largely between studies (Hailwood et al., 1999; Jones et al., 2006; Nabawy et al., 2009; Pfleiderer \& Halls, 1990, 1993, 1994). As the pore fabric defines numerous physical properties of a rock, magnetic pore fabric measurements can provide important predictions e.g. for fluid flow applications. These predictions will be more useful if magnetic pore fabrics can be interpreted quantitatively. A detailed and quantitative understanding of the way magnetic anisotropy reflects mineral and pore fabrics is therefore essential in structural, geodynamic and fluid migration studies employing magnetic fabric methods.

Three physical sources mainly control the magnetic anisotropy carried by the minerals in a rock: (1) magnetocrystalline anisotropy, related to the crystal structure, cation site occupancy, and mineral composition (Biedermann, 2018, and references therein), (2) shape anisotropy, due to selfdemagnetization of strongly magnetic non-equidimensional bodies (Clark \& Emerson, 1999; Osborn, 1945; Stoner, 1945), and (3) distribution anisotropy resulting from magnetostatic interactions between strongly magnetic grains (Hargraves et al., 1991; Stephenson, 1994). The magnetic fabric of the rock is then a superposition of contributions from (1) crystallographic preferred orientation of minerals with magnetocrystalline anisotropy, (2) shape preferred orientation of grains possessing shape anisotropy, and (3) non-random distribution of grains that interact magnetostatically. In strongly magnetic grains with cubic intrinsic anisotropy such as magnetite, shape and distribution anisotropy often outweigh the magnetocrystalline anisotropy. Both shape and distribution anisotropy have been associated with magnetite grains in rocks, and their relative importance is controversially discussed (Cañón-Tapia, 1996, 2001; Grégoire et al., 1998; Grégoire et al., 1995; Hargraves et al., 1991; Stephenson, 1994). Magnetic pore fabrics have traditionally been attributed to shape anisotropy (Hrouda et al., 2000; Pfleiderer \& Halls, 1990, 1993), but recently published results suggest that distribution anisotropy plays an important role too (Biedermann, 2019). Hence, the same two physical sources, shape and distribution anisotropy, are relevant in magnetic pore fabric studies, and to describe the anisotropy carried by magnetite.

This study focuses on the mathematical description of the two types of anisotropy that are relevant to describe and predict the magnetic fabric carried by strongly magnetic grains or impregnated pores: shape anisotropy and distribution anisotropy. A major development towards a quantitative understanding of shape and distribution anisotropy were correlations between images of grains or pores in natural samples and magnetic measurements (Grégoire et al., 1998; Launeau \& Cruden, 1998; Pfleiderer \& Halls, 1993). Models represent a further step towards quantitative mathematical descriptions. Shape anisotropy is modeled based on self-demagnetization of grains, analogously to self-demagnetization of large magnetized bodies (Clark \& Emerson, 1999). Stephenson (1994) developed a model to calculate the distribution anisotropy of equal spherical particles arranged in either infinite lines or infinite planes at constant inter-particle spacing. This model has been adapted to ellipsoidal grains or pores of equal size and shape arranged in infinite lines or planes with equal spacing (Biedermann, 2019; Cañón-Tapia, 1996, 2001). These existing models predict shape and distribution anisotropy in simplified systems with easy geometries, but are not directly applicable to natural rocks. To help solve the controversy on the relative contributions of shape and distribution anisotropy, a model that more closely reflects properties of natural samples is desirable. 
Grains or pores in rocks generally display an irregular distribution, and possess a wide range of sizes, shapes, and orientations. These characteristics of natural rocks were taken into account when developing FinIrrSDA (Figure 1). FinIrrSDA is a Matlab-based code that calculates the Shape and Distribution Anisotropies for all types of particle arrangements, including FINite IRRegular assemblies of particles with different sizes, shapes and orientations. These properties of the new model allow predicting shape and distribution anisotropies in a wide range of rocks, and these predictions will ultimately help solve the ongoing controversy on the relative importance of shape and distribution anisotropies. The size, shape, orientation, and distribution of magnetic particles or impregnated pores varies between rocks, and therefore it is expected that shape and distribution anisotropies contribute in different proportions. Hence, the examples shown here illustrate how FinIrrSDA can be applied to different datasets, but will not solve the controversy once and for all. Rather, FinIrrSDA can evaluate the contributions of shape and distribution anisotropies in a specific rock. FinIrrSDA is conceptualized as a forward model, and requires information on the magnetic particles (position, dimensions, orientation, magnetic properties). As with every forward model, the reliability of the results depends on the quality of the input data; ideally, the input data would contain a full 3D description with perfect resolution and cover a representative volume of the sample, which is not achievable with any existing characterization method. Limited resolution, and the lack of depth information in image data and related uncertainties are discussed, as well as assumptions and potential limitations. Note that each of the examples presented here provides insight on anisotropy contributions in a specific setting, but it is not the purpose of the examples to universally solve a controversy that has been going on for decades. The model predictions and the results presented here apply equally to strongly magnetic grains in rocks and to ferrofluid-filled pores. The generic terms 'body' or 'particle' will be used throughout this manuscript to describe any part of the rock that is strongly magnetic compared to the properties of the bulk rock. In line with its envisaged applications, FinIrrSDA assumes equal intrinsic magnetic susceptibilities for all particles, but could easily be adapted to include sets of particles with variable magnetic properties. 


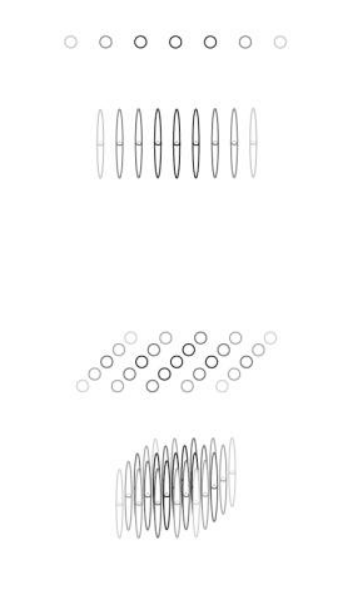

Existing models

Particle properties:

- Equal size and shape

- Same orientation

- Spherical or ellipsoidal

Particle arrangement:

- Regular spacing

- Lines or planes

- Infinite

Magnetostatic interactions:

- Nearest neighbors only

- Dipolar, at particle centers

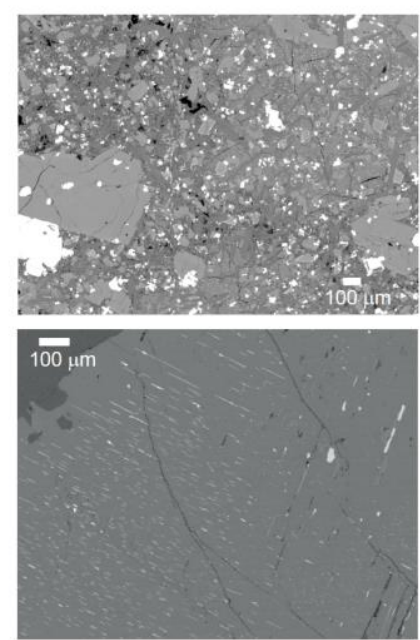

Reality (natural samples)

Particle properties:

- Range of sizes and shapes

- Orientation distribution

- Complex, irregular shapes

Particle arrangement:

- Irregular arrangement

in 3D

- Finite

Magnetostatic interactions:

- All particles

- Spatial variation within particle

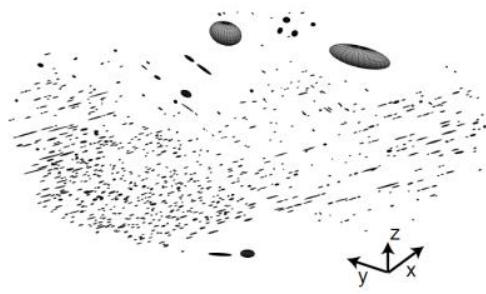

\section{FinlrrSDA}

Particle properties:

- Range of sizes and shapes

- Orientation distribution

- Ellipsoid/cylinder approximation

Particle arrangement:

- Irregular arrangement

in 3D

- Finite

Magnetostatic interactions:

- All particles

- Dipolar, at particle centers

Figure 1: Properties of individual particles, their arrangement, and interactions of existing models compared to typical properties of natural rock samples. Examples for rock samples are backscattered electron images showing a basalt from Cape Verde, and a pyroxene grain in an oxide gabbro from the Duluth Complex, MN, USA. The code FinIrrSDA proposed in this study still relies on approximations with respect to particle shape and the description of magnetic interactions, but closes major gaps between existing models and reality.

\section{Theory}

Shape and distribution anisotropy are observed for strongly magnetic bodies surrounded by nonmagnetic material. Shape anisotropy is based on the effect of self-demagnetization, a result of energy minimization at the outer boundary of a strongly magnetic body (Clark \& Emerson, 1999; Lowrie, 1997). Self-demagnetization can be mathematically described as a secondary demagnetizing field $\vec{H}_{d}=-N \vec{M}$ that depends on the magnetization $\vec{M}$ of the body and its self-demagnetization tensor $N$, defined by body shape (Lowrie, 1997). For a body with intrinsic susceptibility $k_{\text {int }}$, the demagnetizing field reduces the effective field experienced by the body, thus decreasing its observed susceptibility to $k_{\text {obs }}=\left(I+k_{\text {int }} N\right)^{-1} k_{\text {int }}$, where $I$ is the unit matrix (Clark, 2014). The self-demagnetization tensor $N$ is defined exactly for ellipsoids (Osborn, 1945; Stoner, 1945), and can be approximated for other bodies (Sato \& Ishii, 1989). For spherical bodies, $N=\frac{1}{3} I$ (in the SI system of units), and they display no shape anisotropy. Shape anisotropy is particularly pronounced for strongly elongated or flattened particles with strong intrinsic susceptibility. In addition to reducing the magnetization intensity, self-demagnetization and the resulting shape anisotropy generally 
cause deviations of the magnetization direction away from the direction of the externally applied field $\vec{H}_{\text {ext }}$ (Figure 2a).

Distribution anisotropy results from magnetostatic interactions between strongly magnetic particles. The magnetization in each particle generates a secondary magnetic field $\vec{H}_{p, i}, i=1 \ldots n-1$, where $n$ is the number of particles, that acts on the other particles in addition to the externally applied field. Assuming that particle spacings are large enough, the magnetization of particle $i$ can be described as a dipole field acting on all particles $j, j \neq i$. The field strength and direction depend on the interparticle distance $r$ and the relative positions of the particles to each other, described by the angle $\theta$ between the dipole axis and the vector connecting the particles. In the polar coordinate system of the dipole, the secondary field generated by particle $i$ and experienced by particle $j$ can be written as:

$\vec{H}_{p, i j}=\frac{2 m \cos \theta_{i j}}{4 \pi r_{i j}{ }^{3}} \widehat{r_{l j}}+\frac{m \sin \theta_{i j}}{4 \pi r_{i j}{ }^{3}} \widehat{\theta_{l j}}$

where the magnetic moment $\vec{m}=\vec{M} V$, and $V$ is the particle volume (Lowrie, 1997; Tauxe et al., 2018). The effect of the secondary field can decrease or increase the effective field at another particle, depending on the relative positions of the particles and the field direction, and decreases with increasing inter-particle distance (Figure 2b).

Independent of its source, magnetic anisotropy is represented mathematically by a symmetric second-order tensor $\boldsymbol{k}$, or geometrically by an ellipsoid. The eigenvalues of this tensor, $k_{1} \geq k_{2} \geq$ $k_{3}$, are referred to as maximum, intermediate and minimum principal susceptibilities and define the lengths of ellipsoid axes. The corresponding eigenvectors define the principal susceptibility directions, or the orientation of the ellipsoid. Six independent elements are required to fully describe $\boldsymbol{k}$. Common experimental setups measure properties in more than six directions to minimize the effect of measurement noise, and to determine data quality (Jelinek, 1977, 1996). In the absence of noise, six directions are sufficient. 

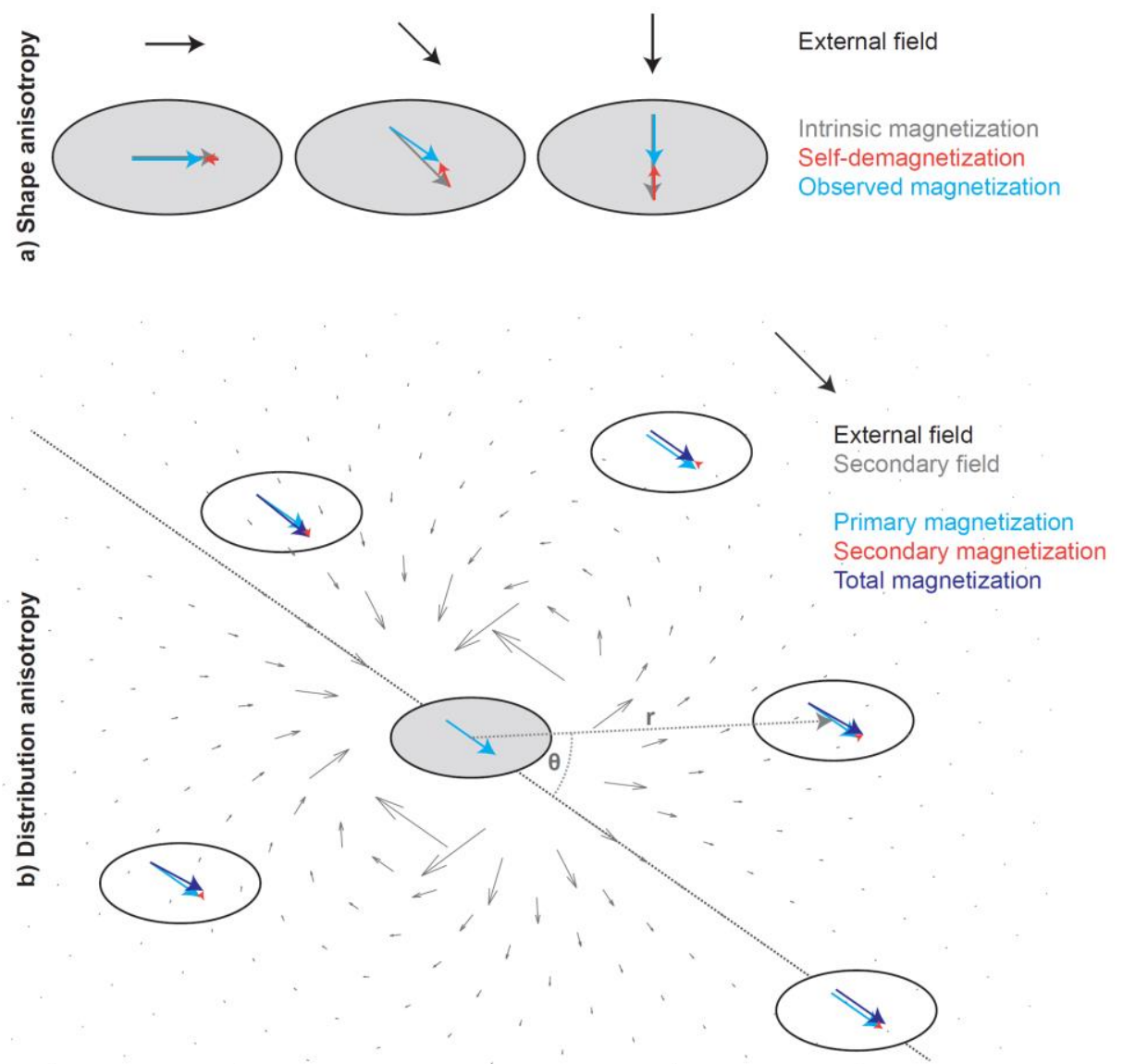

Figure 2: Schematic overview of shape and distribution anisotropies.

\section{Model: Assumptions, applicability and setup}

FinIrrSDA calculates shape and distribution anisotropy for homogeneous ellipsoidal or cylindrical particles in a non-magnetic matrix. It is assumed that the particle magnetization is high, and that particle properties and their preferred orientation are such that magnetocrystalline anisotropy is negligible compared to the combined effect of shape and distribution anisotropies. This assumption is valid for example for elongated magnetite grains in a silicate rock, or for ferrofluid-filled pores in a weakly magnetic sediment.

Self-demagnetization and shape anisotropy are approximated by the magnetic anisotropy of best-fit ellipsoids (or best fit cylinders) for each particle. Thus, the calculated shape anisotropy is most accurate for particles resembling these shapes, but may deviate for complicated irregular grains.

Magnetostatic interactions and secondary fields generated by each particle's magnetization on all other particles are approximated by point source magnetic moments at the center of particle $p_{i}$, assuming that the central secondary fields at particles $p_{j}, j=1 . . n, j \neq i$ are representative for the average field the particles experience. Thus, the model is valid as long as inter-particle distances are large enough, so that the dipole equation adequately represents magnetic interactions, and the secondary field is sufficiently homogeneous throughout the particle. This limitation equally applies to previously published distribution anisotropy models. The distance at which this assumption is valid was determined from a 2-particle system, where the magnetization of particle $p_{1}$, due to an externally applied field $\vec{H}_{\text {ext }}$, gives rise to a secondary field $\vec{H}_{p}$ at the location of particle $p_{2}$, so that 
the total field experienced by $p_{2}$ is $\vec{H}_{\text {tot }}=\vec{H}_{\text {ext }}+\vec{H}_{p} \cdot \vec{H}_{p}$ was calculated at the center of $p_{2}$, as well as by averaging all secondary fields on a regularly spaced grid throughout the volume of $p_{2}$. To simplify the grid average, particles were assumed to be rectangular rods, whose $x$ and $y$ dimension remained constant, while their $z$-dimension varied to form isometric, flattened (oblate) and elongated (prolate) particles. The particle size is given here by the diameter in the $x y$ plane, so that prolate particles have larger volumes than oblate particles. The external field was applied parallel to the particles' $x, y$, and $z$ directions, and $p_{2}$ was shifted along the $x$-axis to various distances from $p_{1}$. Four intrinsic susceptibilities were used, $20 \mathrm{SI}$ and $2.4 \mathrm{SI}$ to test differences in the model results related to the uncertainty in the $k_{\text {int }}$ of magnetite, $1 \mathrm{SI}$ which is close to the intrinsic susceptibility of the ferrofluid used by Jones et al. (2006), and $0.16 \mathrm{SI}$, corresponding to a more strongly diluted ferrofluid (Pugnetti et al., 2020).

The strength of $\vec{H}_{p}$ and its variability throughout $p_{2}$ depends on $p_{1}$ 's magnetization and thus its intrinsic susceptibility, particle geometry and external field direction, as well as the inter-particle spacing (Figure 3). For spherical and prolate particles $p_{2}$, the central field overestimates the average field experienced by the particle. For spherical particles, differences between the central and average fields are independent of field direction, and the overestimation is $\sim 20 \%$ for a particle spacing of 1 , i.e. when the sides of $p_{1}$ and $p_{2}$ are touching, $\sim 10 \%$ at a spacing of 1.3 , and drops below $1 \%$ when the spacing increases to 2.3 particle diameters. The overestimation and its dependence on the direction of $\vec{H}_{\text {ext }}$ become stronger with increasing prolateness of the particles. Conversely, for oblate particles, the central field underestimates the average field experienced by $p_{2}$, except when $\vec{H}_{\text {ext }} / / y$ and inter-particle spacing is small (Figure 3a).

The deviation of the total field experienced by $p_{2}, \vec{H}_{t o t}$, from $\vec{H}_{\text {ext }}$ depends on particle geometry, inter-particle spacing, as well as the intrinsic susceptibility. Deviations are generally larger for smaller spacing, larger $k_{\text {int }}$, and increase from oblate to spherical to prolate geometries (Figure $3 \mathrm{~b}$ ). For the arrangement of $p_{1}$ and $p_{2}$ along the $x$-axis as investigated here, $\left|\vec{H}_{\text {tot }}\right|>\left|\vec{H}_{\text {ext }}\right|$ when the external field is applied along $x$, and $\left|\vec{H}_{\text {tot }}\right|<\left|\vec{H}_{\text {ext }}\right|$ when the applied field was along $y$ or $z$. The difference between $\left|\vec{H}_{\text {tot }}\right|$ and $\left|\vec{H}_{\text {ext }}\right|$ at the particle center drops to $1 \%$ of the external field at $\sim 4$ particle diameters spacing for strongly prolate particles and $k_{\text {int }}=2.4$. Averaged over $p_{2},\left|\vec{H}_{\text {tot }}\right|$ is within $1 \%$ of $\left|\vec{H}_{\text {ext }}\right|$ at a spacing of 2 (oblate particles) to 8 (prolate particles) particle diameters for $k_{\text {int }}=20$, and at smaller distances for weaker $k_{\text {int }}$. Figure $3 \mathrm{~b}$ also shows that $\left|\vec{H}_{\text {tot }}\right|$ deviates less than $0.1 \%$ from $\left|\vec{H}_{\text {ext }}\right|$ at spacings of ca 4, 6 and $>10$ particle diameters for strongly oblate, spherical, and strongly prolate particles, respectively $\left(k_{i n t}=20\right)$. These distances may be taken as threshold values at which the influence of magnetostatic interactions becomes negligible. However, note that although a $1 \%$ or $0.1 \%$ deviation in field intensity may seem small, these results apply to the onesided effect of $p_{1}$ on $p_{2}$, and the effect of magnetostatic interactions will add up when the number of particles increases, and their mutual effects on each other are considered.

Additionally, the secondary field is anisotropic, so that effects on the anisotropy of the apparent susceptibility prevail at larger particle spacing, especially when $k_{\text {int }}$ is large. For example, a $1 \%$ change in anisotropy is expected from a one-sided interaction of one particle on a strongly prolate particle at a spacing of ca 6 particle diameters for $k_{\text {int }}=2.4$ and $>10$ particle diameters for $k_{\text {int }}=20$ (Figure 3c). The results shown in Figure 3 indicate that the largest errors in estimating the effect of magnetostatic interactions are made for side-by-side configurations of closely spaced and strongly magnetic prolate particles. Unfortunately, closely spaced and strongly magnetic particles are also those with the highest expected contribution to distribution anisotropy, so that care needs to be 
taken that the model is applied to particle assemblages with large enough spacing to avoid artefacts due to the differences between central and average particle fields. The particles in many natural rock samples are less anisotropic or at a larger spacing than what is used in these extreme cases to explore the model limitations. However, some rocks will contain side-by-side configurations of needle-shaped particles, or particles close together, and for those it is important to be aware of FinlrrSDA's limitations. A solution would be to compute the variation of magnetization throughout the particle rather than solely at the particle center, if the computational power is available. It computational power is limited, the data presented in Figure 3 can help quantify the expected uncertainties.

Within the limits of the assumptions outlined above, FinIrrSDA is aimed to be as broadly applicable as possible. In particular, it was designed to calculate magnetic anisotropy for finite and irregular distributions of particles with unequal dimensions, shapes, and orientations. The calculations presented in this study assume equal intrinsic susceptibility for each particle, but the model could be easily adapted to incorporate individually defined susceptibilities for each particle. The following parameters serve as input for the model:

(1) $x, y$, and $z$ coordinates of the particle centers

(2) lengths of the major, intermediate and minor axes of best-fit ellipsoids

(3) orientation vectors (defined by their declination and inclination) of the ellipsoid axes

(4) intrinsic magnetic susceptibility

The input data reflecting the particle properties, (1) to (3) are read in as a table where each row represents one particle. The particle data could be the known structure of synthetic samples, or derived from tomography scans, or images. The quality of the particle data in terms of accuracy, representativeness and resolution is an important factor that determines the reliability of the modeled anisotropies. 3D representations of the particles are preferable, but 2D image analysis data can be used subject to certain assumptions. Spatial resolution can typically be increased by analyzing smaller samples, and often a compromise between resolution and representativeness will have to be made. Similarly, 2D images may have higher resolution than 3D datasets, at the expense of depth information.

3D particle distributions can be characterized by X-ray computed tomography (XRCT), with typical voxel resolutions on the order of $\sim 1-10 \mu \mathrm{m}$, and several voxels necessary to describe the particle shapes and orientations (Baker et al., 2012; Ketcham, 2005; Landis \& Keane, 2010). Given that isometric SD and PSD magnetite grains have typical sizes of $50 \mathrm{~nm}-1 \mu \mathrm{m}$ and $1-20 \mu \mathrm{m}$, respectively (Clark, 1997, Table 4; larger threshold values for elongated particles), mainly MD grains will be included in XRCT-derived input data. Higher-resolution input data that also includes SD and PSD grains will need to be treated with special care, because SD magnetite causes inverse low-field magnetic fabrics (Rochette et al., 1999; Rochette et al., 1992), and the anisotropy of PSD grains and its mathematical description still need to be defined.

When studying pore fabrics, the intrinsic susceptibility is controlled by the ferrofluid used for impregnation and its concentration. Estimating the intrinsic susceptibility of strongly magnetic materials such as magnetite is challenging, because $k_{o b s}=\left(I+k_{\text {int }} N\right)^{-1} k_{\text {int }}$ approaches $k_{o b s}=$ $N^{-1}$ for large $k_{\text {int }}$. Therefore, the observed magnetic susceptibility in spherical MD magnetite is $k_{o b s} \sim 3$. For SD grains, $k_{o b s}$ depends on the grain shape, and varies between 1.33 (needle-shaped grains) to $20 \mathrm{SI}$ (equidimensional grains with magnetocrystalline anisotropy) (Dunlop \& Özdemir, 
1997; Stacey \& Banerjee, 1974). Typical susceptibility values for SD magnetite of 2.4 SI and 1.3-12.6 $\mathrm{SI}$, and for MD magnetite of 2.8 SI and 2.5-3.8 SI, as reported by (Thompson \& Oldfield (1986, Table 4.2 ) and Clark (1997, Figure 3) respectively, agree with these theoretical limits. Intrinsic magnetite susceptibilities vary largely depending on grain size and domain state, crystallinity and dislocation density, and Clark (1997) reported values from 13 SI (fine-grained, poorly crystalline, stressed or inhomogeneous grains) to $130 \mathrm{SI}$ (coarse and well-crystallized grains) and identified a 'typical value' of $20 \mathrm{SI}$. The large uncertainty in $k_{\text {int }}$ leads to uncertainties in the models results, and this is tested by calculating models for $k_{\text {int }}$ values of $2.4 \mathrm{SI}$ as well as $20 \mathrm{SI}$. 
a) 2-particle system: setup and secondary field due to $p_{1}$ at center of and averaged over $p_{2}$
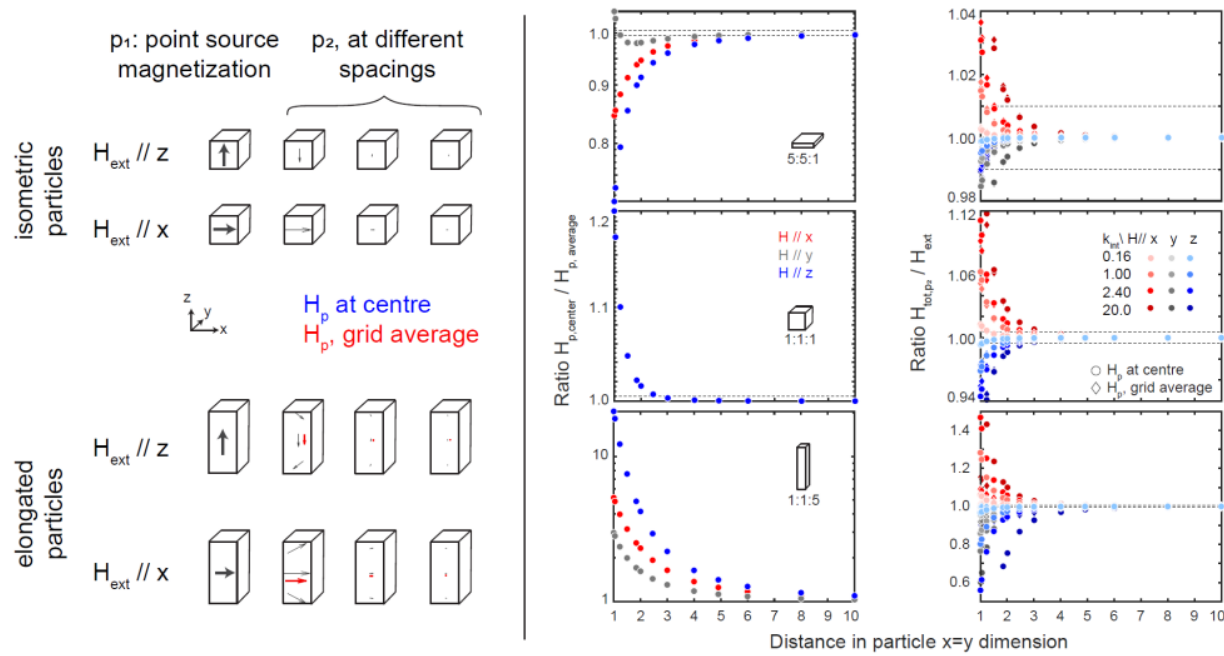

b) Distance at which the intensity of $\mathrm{H}_{\mathrm{p}}$ is $1 \%$ and $0.1 \%$ of $\mathrm{H}_{\mathrm{B}}$
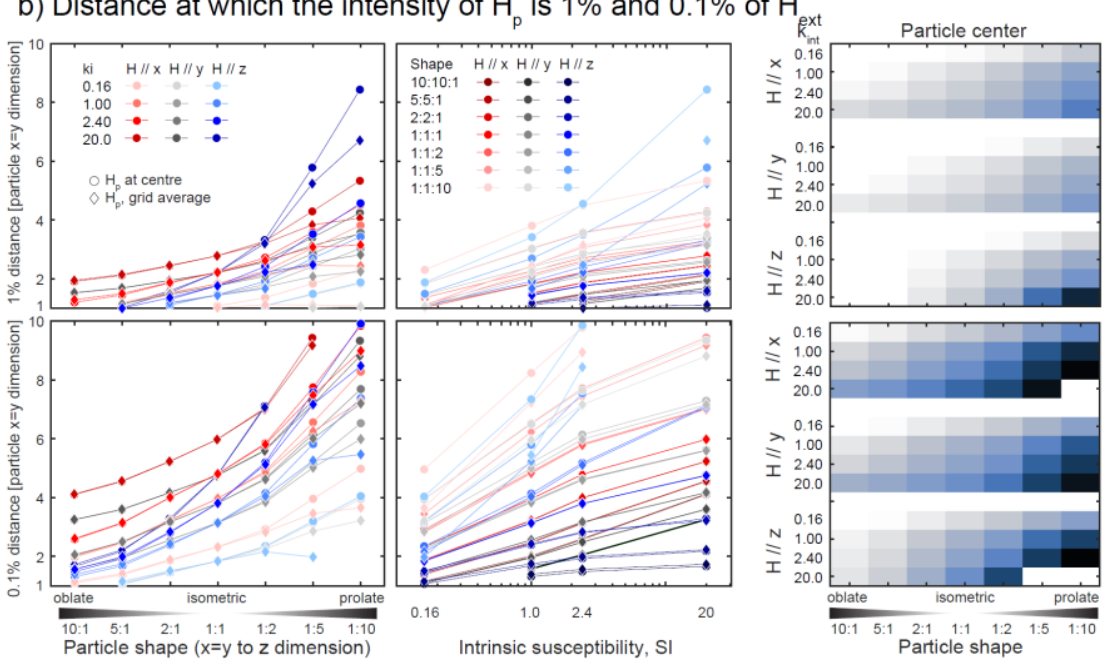

Averaged over entie paricie
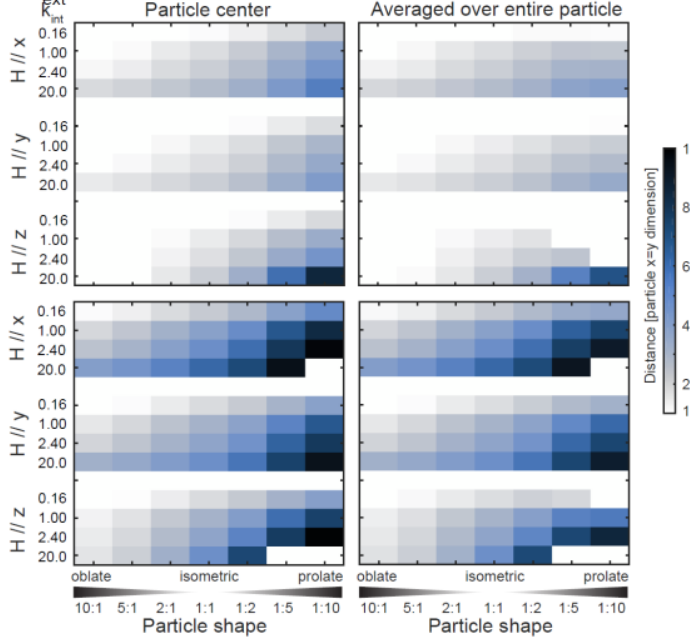

c) Anisotropy of $\mathrm{H}_{\mathrm{p}}$ experienced by $\mathrm{p}_{2}$
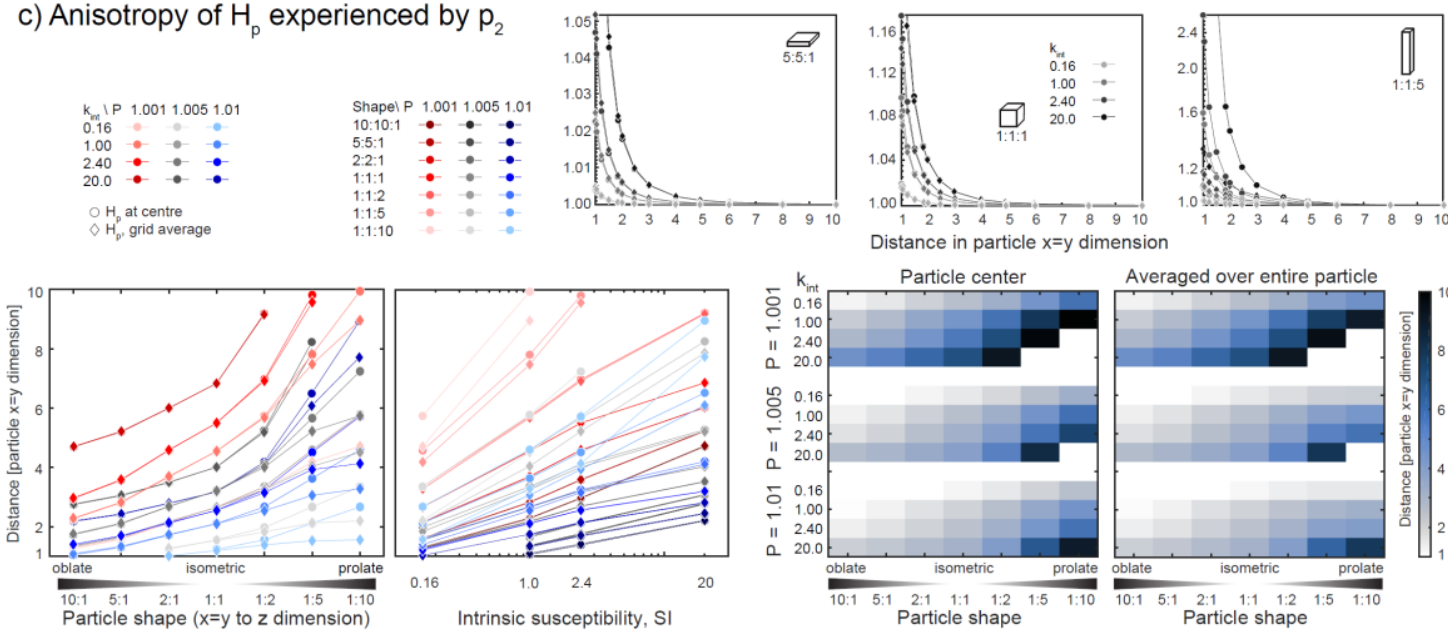

Figure 3: (a) Magnetic interactions in a 2-particle system. The magnetization in particle $p_{1}$ is defined by the external field and the particle's intrinsic susceptibility and shape anisotropy. The field experienced by particle $p_{2}$ is a superposition of the external field $\vec{H}_{\text {ext }}$ and the secondary field $\vec{H}_{p}$ created by $p_{1} . \vec{H}_{p}$ is not homogeneous throughout $p_{2}$, especially for large and strongly elongated particles at small interparticle spacings. (b) Distance at which the secondary field is $<1 \%$ of $\vec{H}_{\text {ext }}$ as a 
function of intrinsic susceptibility and particle geometry. (c) Anisotropy of the secondary field. Perceptually uniform color-maps are used to prevent visual distortion of the data (Crameri, 2018).

To calculate magnetic anisotropy of the particle assemblage, FinIrrSDA (1) determines the selfdemagnetization and $k_{o b s}$ tensors for each particle, (2) based on $k_{o b s}$ calculates the primary magnetization of each particle for a series of 6 independent directions of $\vec{H}_{\text {ext }}$, (3) computes the vector sum of secondary fields $\vec{H}_{p, i}$ experienced by particle $i$ due to all other particles for every direction of $\vec{H}_{\text {ext }}$, and (4) predicts the total magnetization of each particle taking into account the secondary fields and the particle's shape anisotropy for each direction of the external field. The magnetostatic interactions (step (3)) change the total field experienced by each particle. This in turn affects the particles' magnetization, and their secondary field that adds to the total effective field experienced by all other particles, in particular those close-by. Hence, a second iteration of steps (2) to (4) is performed when $\left|\vec{H}_{t o t}\right|$ of a particle differs more than $1 \%$ from $\left|\vec{H}_{\text {ext }}\right|$, or the directions of $\vec{H}_{t o t}$ and $\vec{H}_{\text {ext }}$ deviate by more than $1^{\circ}$ from each other. The integrated shape anisotropy of all particles is calculated after step (1), by adding the $k_{o b s}$ tensors, weighted by the particle volume calculated from the lengths of the axes. The combined shape and distribution anisotropy tensor is calculated after step (4) has completed for all field directions. Magnetization components parallel to $\vec{H}_{\text {ext }}$ define the anisotropy tensor. Both results are given as susceptibility normalized by total particle volume. Two distinct coordinate systems are employed for different calculation steps in the model. Shape anisotropy, magnetization directions, and final anisotropy tensors are computed in the sample ( $x y z)$ coordinate system. Conversely, the magnetic interactions are computed in the coordinate system of the dipole generating $\vec{H}_{p}$, defined by the magnetization direction of the particle after shape anisotropy is taken into account. The latter are then transformed back to the sample coordinate system. An overview of the code including the used coordinate systems is given in Figure 4.

The model was tested for self-consistency, i.e. whether anisotropy tensors are the same when the entire particle assembly is rotated with respect to the coordinate system. Additional tests verified that calculations are independent of whether the 3 axes of a particle define a right-handed or lefthanded coordinate system. Finally, FinIrrSDA models of finite lines and planes consisting of different numbers of particles were compared to results of published models for infinite lines or planes of regularly spaced equal particles (Biedermann, 2019; Cañón-Tapia, 1996; Stephenson, 1994). Input parameters for these models were (1) the particle dimensions along their main axes, and (2) the spacing between particles. For both line and plane models, the particles were rotationally symmetric with their symmetry axes along $z$, and particles aligned on the $x$-axis or in the xy plane for linear and planar arrangements, respectively. For the finite models, the total extension of the lines is defined as $(N-1) * d$, where $N$ describes the number of particles, and $d$ the spacing between two neighboring particles along the $x$-axis. Finite plane models were computed for an equal number of particles along $\mathrm{x}$ and $\mathrm{y}$, and the extension of the plane is $(\sqrt{N}-1) * d$ or $\left(N_{x}-1\right) * d$, where $N=$ $N_{x} N_{y}$ is the total number of particles, and $N_{x}=N_{y}$ are the number of particles along the $x$ and $y$ axes. These finite models were compared to models on infinite lines or planes of the same particles and the same spacing (Figure 5).

The comparison between FinIrrSDA (finite lines and planes, interactions between all particles) and previous models (infinite lines and planes, nearest neighbor interactions only) for the same particle spacing shows considerable differences, especially when the number of particles is small. The anisotropy of a small number of particles is more strongly dominated by shape anisotropy, while the contribution of distribution anisotropy is weak due to the small number of interaction partners. As 
the number of particles increases, the number of interactions increases, thus leading to stronger influence of distribution anisotropy. The difference in distribution anisotropy and thus total anisotropy is large when the line increases from $N=1$ to $N=2$ and from $N=2$ to $N=3$, whereas the influence of one additional particle is minimal when $\mathrm{N}$ changes from e.g. 100 to 101. Directional susceptibilities and anisotropy calculated by FinIrrSDA reflect these changes in the magnetic interactions, whereas the previously used infinite models provide a single estimate independent of the number of particles in the assembly. Therefore, largest deviations are observed for small $\mathrm{N}$, where the finite models show a gradual change in anisotropy from the shape-anisotropy-dominated single particle to assemblies of several particles. At large numbers of particles, the line models shown in Figure 5 reach more or less constant directional susceptibilities, which however, deviate from those of the infinite models. The reason for this is that FinIrrSDA considers interactions between all particles, whereas the previous models only considered nearest-neighbor-interactions. Therefore, the effect of distribution anisotropy is stronger in FinIrrSDA, and more realistic. Also for planar particle assemblies, FinIrrSDA shows a gradual transition for a single particle controlled purely by shape anisotropy to a plane of $2 * 2,3 * 3, n * n$ particles, i.e., with increasing influence of distribution anisotropy. The differences between finite and infinite models of high particle numbers with a given particle spacing $d$ are generally larger for planar assemblies compared to linear assemblies, due to the increased number of non-nearest neighbors within a given radius. For the same reason, larger differences in directional susceptibility and magnetic anisotropy are predicted between planar assemblies with $10 * 10=100$ particles compared to planar assemblies of $11 * 11=$ 121 particles, whereas smaller differences are expected between lines of 100 vs 121 particles. Therefore, the errors made by predictions based on the previously available infinite models is larger for 2D (planar) assemblies of magnetic particles than for 1D (linear) ones. Because iron oxides and impregnated pores in natural rocks are arranged in 3D, even larger uncertainties would be expected, if the infinite models were able to predict the distribution anisotropy for that geometry. Hence, the application of finite models taking into account all interactions between all particles is especially important when these particles are arranged in 3D.

As $d$ decreases, interactions become stronger, and distribution anisotropy will be more prominent. Strong interactions lead to changes in the effective fields and magnetizations experienced by each particle may change, and FinIrrSDA takes this into account by a second iteration step. In the examples shown in Figure 5, a second iteration was only necessary for the prolate particles. The change in predicted susceptibility after the second iteration is larger for the planar arrangement than the linear arrangement, again because the number of particles within a given radius and therefore the secondary field due to interactions is larger in the planar arrangement. Both the infinite and finite models show a stronger distribution anisotropy component when particles are closer together. The differences of FinlrrSDA to previously published models are largest for small $d$, where the interactions are stronger (Figure $5 \mathrm{~d}$ ).

Model run times depend on the computational power as well as the properties of the particle assemblage. Typical run times on a standard laptop (Intel ${ }^{\circledR}$ Core $^{\mathrm{TM}}$ i7-7500 CPU @ $2.7 \mathrm{GHz}$ processor, 16 GB RAM) are < $0.5 \mathrm{~s}$ for models with < 10 particles, 1 s for 50 particles, 2-3 s for 100 particles, and slightly more than $1 \mathrm{~min}$ for 1000 particles. The largest model shown here contains $\sim 20000$ particles and the calculation took ca. 1-2 days. 


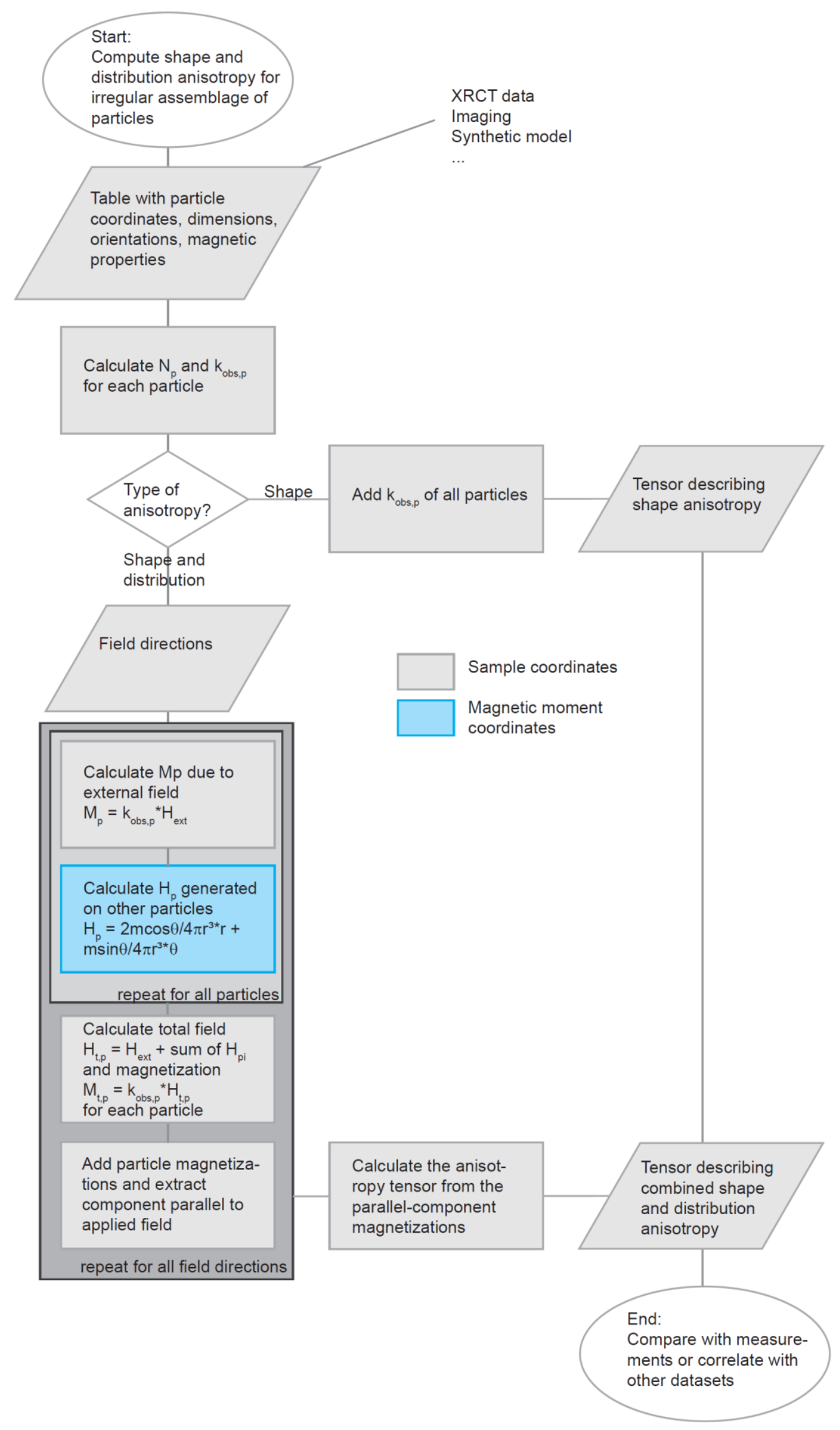

Figure 4: Schematic overview of the FinIrrSDA model. 

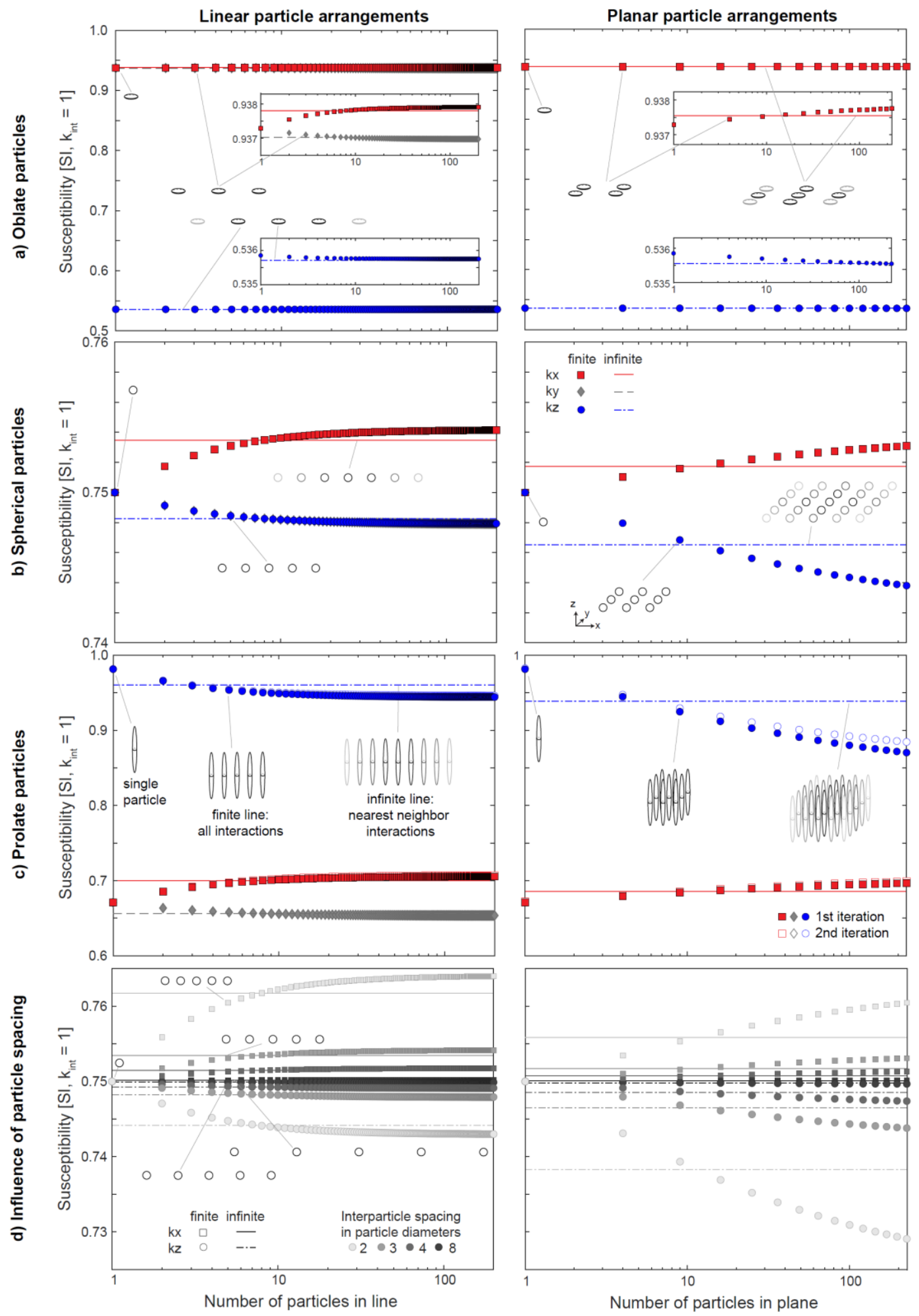

Figure 5: Differences between FinlrrSDA and previously published infinite models. An intrinsic susceptibility of 1 (SI) was used, and results are shown for three particle shapes (strongly oblate ellipsoids (a), spheres (b), and strongly prolate ellipsoids (c)) in either linear or planar arrangements, and a spacing of three times the particle diameter in the xy plane. (d) Influence of interparticle spacing on the example of spherical particles. Symbols indicate the results of finite particle assemblages with all interactions taken into account, and lines reflect infinite models considering nearest-neighbor-interactions only. 


\section{Applications}

The FinIrrSDA model is universally applicable to datasets from which particle dimensions and orientations can be extracted, as long as the particle data is representative of the sample volume, and has sufficient resolution. To illustrate the versatility of the model, two examples will be shown here, (1) computing the expected magnetic pore fabric from the known 3D pore structure of synthetic samples, and (2) approximating the magnetic anisotropy in a plane based on BSE images. All model results are verified against experimental data. In both cases, the model provides information on the relative contributions of shape and distribution anisotropies, and defines which is more important in that specific dataset. However, the main goal of the examples is to highlight the abilities and limitations of the FinIrrSDA model.

\subsection{D: Magnetic pore fabrics in synthetic samples with defined pore geometries}

The first example is a 3D model of the magnetic anisotropy caused by ferrofluid-filled cavities in synthetic samples. The pore shapes and geometries used here reflect the synthetic samples and measurements by Jones et al. (2006), made to study bedding-, capillary- and crack-like pore fabrics. The pores in these synthetic samples are cylinders of known length and diameter, arranged in lines or planes with known geometry, but undefined distance between the cavities. The intrinsic susceptibility of the ferrofluid they used was 1.09 (SI), and they characterized the magnetic anisotropy by the ratio of maximum to intermediate principal susceptibility (magnetic lineation, $L=$ $k_{1} / k_{2}$ ), and intermediate to minimum magnetic susceptibility (magnetic foliation, $\left.F=k_{2} / k_{3}\right)$. The bedding- and capillary-type fabrics consisted of prolate cylinders, while the crack-like fabric was approximated by oblate cylinders. Magnetic anisotropy measurements revealed fabrics resembling the shapes of the individual cavities for the capillary $(F=1, L>1$, corresponding to prolate cylinders) and crack-like ( $F>1, L=1$, corresponding to oblate cylinders) fabrics. Conversely, the prolate cylinders of the bedding-type fabric give rise to a magnetic anisotropy with both $F>1$ and $L>1$ (Jones et al., 2006). The latter observation cannot be explained by shape anisotropy alone and highlights the importance of distribution anisotropy.

Biedermann (2019) calculated shape and distribution anisotropy models for a range of pore spacings, constrained by the sample size, pore size, and number of pores. That study assumed infinite lines or planes and considered only nearest-neighbor interactions. In contrast, finite lines and planes are used in this study, and each of the pores interacts with all other pores in the same sample. Results obtained with FinIrrSDA reflect Jones et al. (2006)'s measurements better than the previous infinite model, in particular for the bedding-like fabric (Figure 6). A comparison between the modeled shape anisotropy and the modeled combination of shape and distribution anisotropies clearly indicates that distribution anisotropy has an important effect on the measurements.

Additionally, a significant improvement from infinite to finite models is observed. In particular, the infinite models show a conceivably better fit of ellipsoidal pores with the measured data, which is in contradiction to the known geometry of the pores in that sample. In contrast, the finite models show a close match between cylindrical pore models and measurements, in agreement with the known sample geometry. Differences between the ellipsoidal and cylindrical pore models highlight the importance of expanding modeling capabilities to non-ellipsoidal particles. The FinIrrSDA results suggest that the distance from pore to pore in the bedding-like fabric of Jones et al. (2006) was $\sim 4.5$ $\mathrm{mm}$. The models for capillary- and crack-like fabrics yield unrealistic anisotropies for small distances, probably related to the assumption of dipolar interactions and central field approximation not being valid for large pores at small distances to each other. 

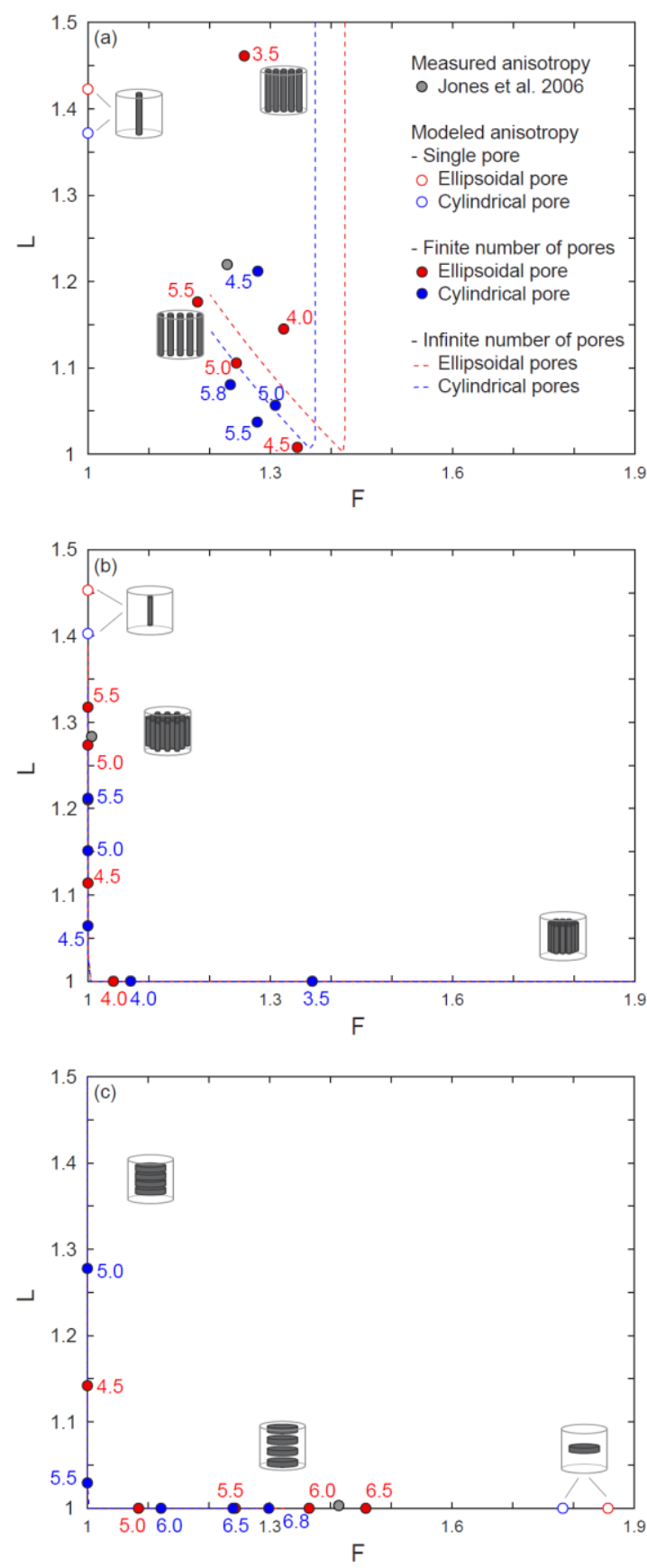

Figure 6: Comparison of FinIrrSDA to the measurements by Jones et al. (2006), and the model by Biedermann (2019) for bedding (a), capillary (b) and crack-like fabrics (c). Dashed lines show changes in results of the infinite models with pore spacing. Numbers next to the data points indicate interpore distances for FinIrrSDA in $\mathrm{mm}$. 


\subsection{D: In-plane anisotropy of an oxide gabbro}

In the second example, the magnetic anisotropy arising from magnetite in an oxide gabbro from the Duluth Complex, MN, USA in a single plane was determined based on backscattered electron (BSE) images. The gabbro contains two types of magnetite, individual grains and pyroxene-hosted exsolutions. The orientation of the exsolutions in pyroxenes is crystallographically controlled, typically with two groups of exsolutions sub-parallel to the [001] and [100] directions of the pyroxene (Bown \& Gay, 1959; Feinberg et al., 2004; Fleet et al., 1980), leading to an oblate ferromagnetic fabric with the minimum susceptibility parallel to the clinopyroxene [010] axis (Biedermann et al., 2015; Hirt \& Biedermann, 2019).

The BSE images were obtained on a JEOL JXA-8900R electron microprobe at the Department of Earth Sciences, University of Minnesota, MN, USA. A large part of the core surface was imaged using comboscans, identifying a total of 20397 oxide particles (Figure 7a). To save computation time and avoid artefacts due to the image dimensions on the distribution anisotropy calculation, a circular section was cut from the original BSE image (Figure $7 \mathrm{~b}$ ). Oxide particles were isolated by greyscale thresholding, and best-fit ellipses obtained using ImageJ (Figure 7c-7e) (Schneider et al., 2012). Particle analysis provided the orientation distribution of the best-fit ellipses, as well as correlations between aspect ratio or grain orientation with grain size (Figure 7f-h). 2546 oxide particles were identified in the circular section shown in Figure 7b. Image analysis results are limited by resolution; i.e. small oxides occupying a single pixel appear circular, and their orientation angle is 0 . Similarly, the exact shape and orientation cannot be determined for oxides that occupy $2 \times 1$ or $1 \times 2$ pixels. Of the identified oxide particles, 982 were non-circular, and 849 of the non-circular particles showed angles different from $n * 45^{\circ}$. Similar resolution-related considerations are also necessary for 3D input data.

Because self-demagnetization operates in 3D but images provide 2D information on the geometry and distribution of oxides only, certain assumptions were necessary prior to anisotropy modeling: Ellipsoids were constructed by assuming rotational symmetry around the long axes of the fitted ellipses (Figure 7i), which then served as input data for FinIrrSDA. This is a reasonable approximation for needle-shaped exsolutions oriented with their long axis in the viewing plane, but inadequate for e.g. platelets or when needle-shaped exsolutions have their longest axis at a large angle to the viewing plane. Note that circular ellipses, reflecting either grains with truly circular cross sections, or grains not resolved adequately, were transformed to spherical grains. These do not possess shape anisotropy, but contribute to distribution anisotropy.

For the modeling, all oxides identified in the image were assumed to be magnetite. Models were calculated for intrinsic susceptibilities of 2.4 (SI) and 20 (SI), to also quantify the effects of the uncertainty in magnetite susceptibility. Due to the high resolution of the image and the strongly elongated grains, some of these may be in the SD/PSD range, and thus give rise to inverse low-field AMS. Because of this, model results were compared to both low-field AMS and AARM measurements, where the latter is not affected by inverse anisotropy. Model calculations were performed for (1) solely shape anisotropy, and (2) shape and distribution anisotropy in the plane of the image. First calculations were performed on the 2546 particles in the circular section. A second model only included the non-circular particles $(n=982)$, and a third model excluded particles whose orientation could not be resolved $(n=849)$ (Figure $7 d-k$; Table 1). Models calculated from the circular section gave similar results, and appeared strongly controlled by a small number of large particles, which were excluded in a fourth model $(n=843)$. An additional model was calculated including all 20397 particles in the entire BSE comboscan. For the models using $k_{\text {int }}=20 \mathrm{SI}$, the strong modeled Intrinsic magnetization of the largest grains causes unrealistically high interaction 
fields, resulting in large angles between external and effective fields experienced by the other particles. These models show the limitation of the approach for strongly magnetic large particles at small spacing (cf Figure 3) and were not interpreted. For the successful models, only small differences were observed between pure shape anisotropy and the combination of shape and distribution anisotropies.

All models have their minimum susceptibility normal to the image plane, an artefact of the 2D nature of the input data. The anisotropy is lineation-dominated, a direct consequence of the assumption that the long axes seen in the image are symmetry axes of needle-shaped particles. The differences between models for solely shape anisotropy and models including both shape and distribution anisotropy are small with their principal directions agreeing within $<1^{\circ}$, independent of the intrinsic susceptibility used. Comparing the modeled directions for both intrinsic susceptibilities also shows a close agreement with $<2^{\circ}$ deviation. The models with stronger intrinsic susceptibility show larger susceptibilities and anisotropy degrees. For all models, taking into account distribution anisotropy leads to a slightly larger degree of anisotropy (difference of $0.003-0.012$ for $k_{\text {int }}=2.4$, and $0.014-0.080$ for $k_{\text {int }}=20$ ) and a stronger foliation component (difference of $0.001-0.009$ for $k_{\text {int }}=2.4$ and $0.006-0.016$ for $k_{\text {int }}=20$ ), related to the particles being arranged in a plane. The fact that these differences are small indicates that for this sample, the contribution of distribution anisotropy is negligible compared to shape anisotropy.

Larger differences are observed depending on the number of particles included. The first three models give similar results in terms of fabric orientation, degree and shape of the anisotropy. The fourth and fifth model show a second set of fabric orientations, which differ from the first three models. This can be explained by the strong influence of a small number of large grains masking the anisotropy of the aligned smaller grains in the first three models. Only a small number of large grains are present throughout the entire BSE comboscan. Nevertheless, they dominate volumetrically in the circular section. Their influence on the overall anisotropy is reduced when more particles are included. The smaller grains have similar orientation throughout the BSE comboscan and hence dominate the anisotropy when larger parts of the sample are considered in the model.

Table 1: Model results based on particle analysis from BSE images. Number of particles included in each model, computation time (seconds), modeled principal susceptibilities and their orientations and anisotropy parameters for models including shape anisotropy or the combination of shape and distribution anisotropy. All models calculated for intrinsic susceptibilities of $2.4 \mathrm{SI}$ and $20 \mathrm{SI}$. 


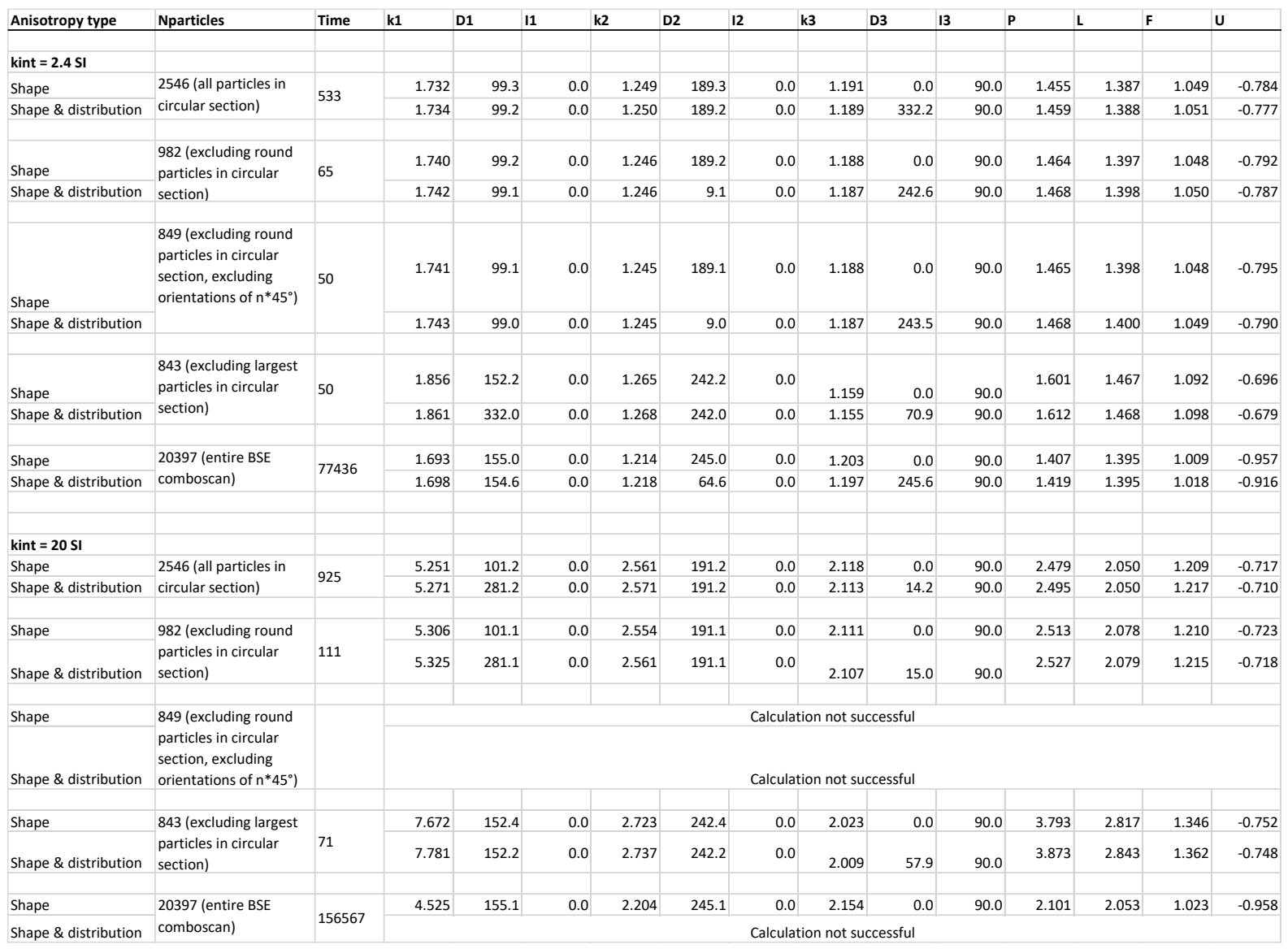

These model results were compared to anisotropy of magnetic susceptibility (AMS) and anisotropy of (partial) anhysteretic remanent magnetization ( $A(p) A R M)$ measured previously (Biedermann, Jackson, Bilardello, et al., 2020; Biedermann, Jackson, Stillinger, et al., 2020). The measurements describe orientations of different subpopulations of grains; AMS being an integrated measure of the alignment of all minerals in the rock, including ferromagnetic and para-/diamagnetic contributions, and potentially subject to an inverse fabric contribution of SD magnetite, and A(p)ARM targeting remanence-carrying minerals in specific coercivity windows, without the complexity of inverse fabrics. Coercivity depends on mineralogy, grain shape and size. Assuming all oxides are magnetite, small and strongly elongated grains will have higher coercivities than large and isometric grains. The measured anisotropies are full second-order tensors, and oriented at angles to the specimen's $x-, y$ and $z$-axes. The maximum susceptibilities are grouped at a large angle to the $x y$ plane on which the BSE image was taken. In order to compare the 3D measurements with the 2D model, the intersection of the magnitude ellipsoid and the image plane was calculated. A comparison of these intersection ellipses with the modeled anisotropy in the image plane is shown in Figure 71 . While the measured and modeled anisotropy in the image plane do not coincide, the agreement is reasonable considering the limitations with the approach: (1) only one plane was imaged and modeled, which may be representative of the alignment of small oxides, but is likely not representative for the larger oxides; (2) the image provides $2 \mathrm{D}$ information only, and assumptions had to be made about the particles' geometry in the third dimension; (3) the smallest particles may not have been imaged due to imperfect resolution; (4) some slightly larger particles occupying a few pixels were imaged, but 
their exact shape and orientation could not be determined; (5) the model assumes that all identified oxide particles have the same composition and magnetic properties, and the intrinsic susceptibility of magnetite had to be assumed; (6) the model considers the contribution of the oxides only, assuming they contribute to the anisotropy proportionally to their size, whereas AMS measurements include contributions from para-/diamagnetic minerals as well as oxides and show inverse fabrics for SD grains, and A(p)ARM measurements take into account only sub-populations of remanencecarrying oxides with a given coercivity. Both models and measurements indicate different anisotropy orientation for small vs large oxide particles; the model calculated for small particles best reflects the ApARM ${ }_{50-100}$ and $A_{p A R M} 100-180$ which target high-coercivity magnetite, i.e., elongated small particles. The models dominated by large particles seemingly reflect the AMS and low-coercivity A(p)ARMs; however, this resemblance should be interpreted with caution given the statistical limitations related to the small number of large particles that were imaged. In any case, the anisotropy in this sample is dominated by shape anisotropy, while distribution anisotropy is negligible.

Despite the limitations, this example illustrates how certain anisotropy predictions can be made from a $2 \mathrm{D}$ image. Nevertheless, 3D imaging techniques, provided their resolution is large enough, provide a better input for the model. 

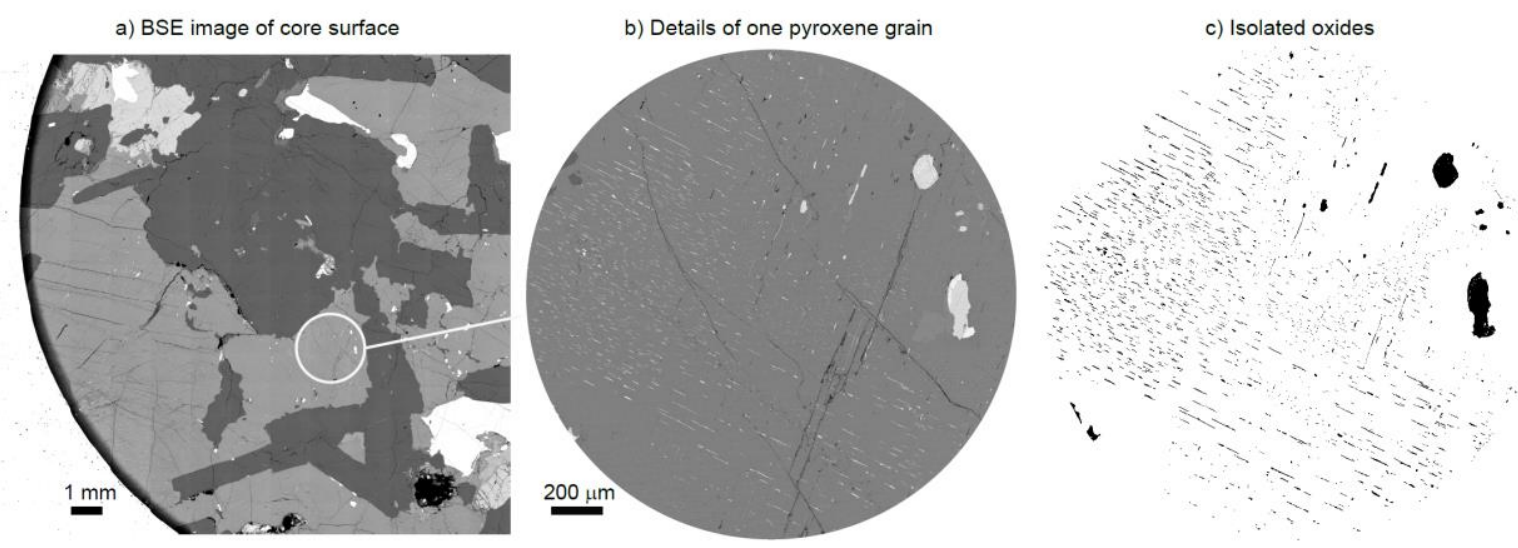

d) Best-fit ellipses, all oxides

e) Subgroups for image analysis and models

f) Orientation distributions

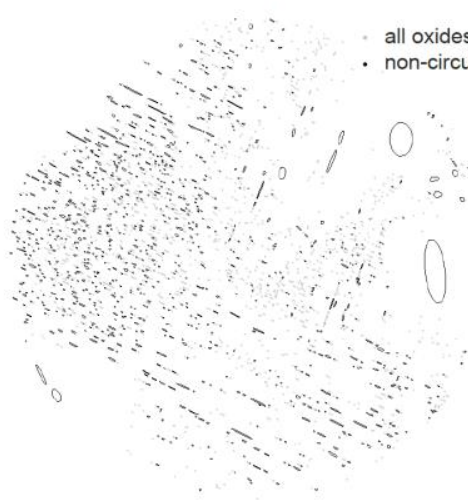

g) Aspect ratio vs size

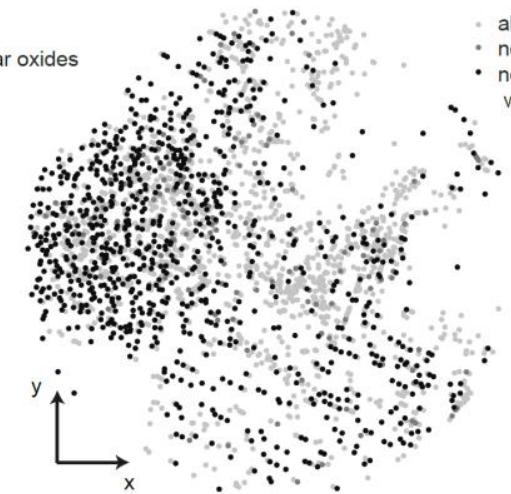

all oxides
non-circular oxides 200

non-circular oxides

w/o angles $n^{\star} 45$
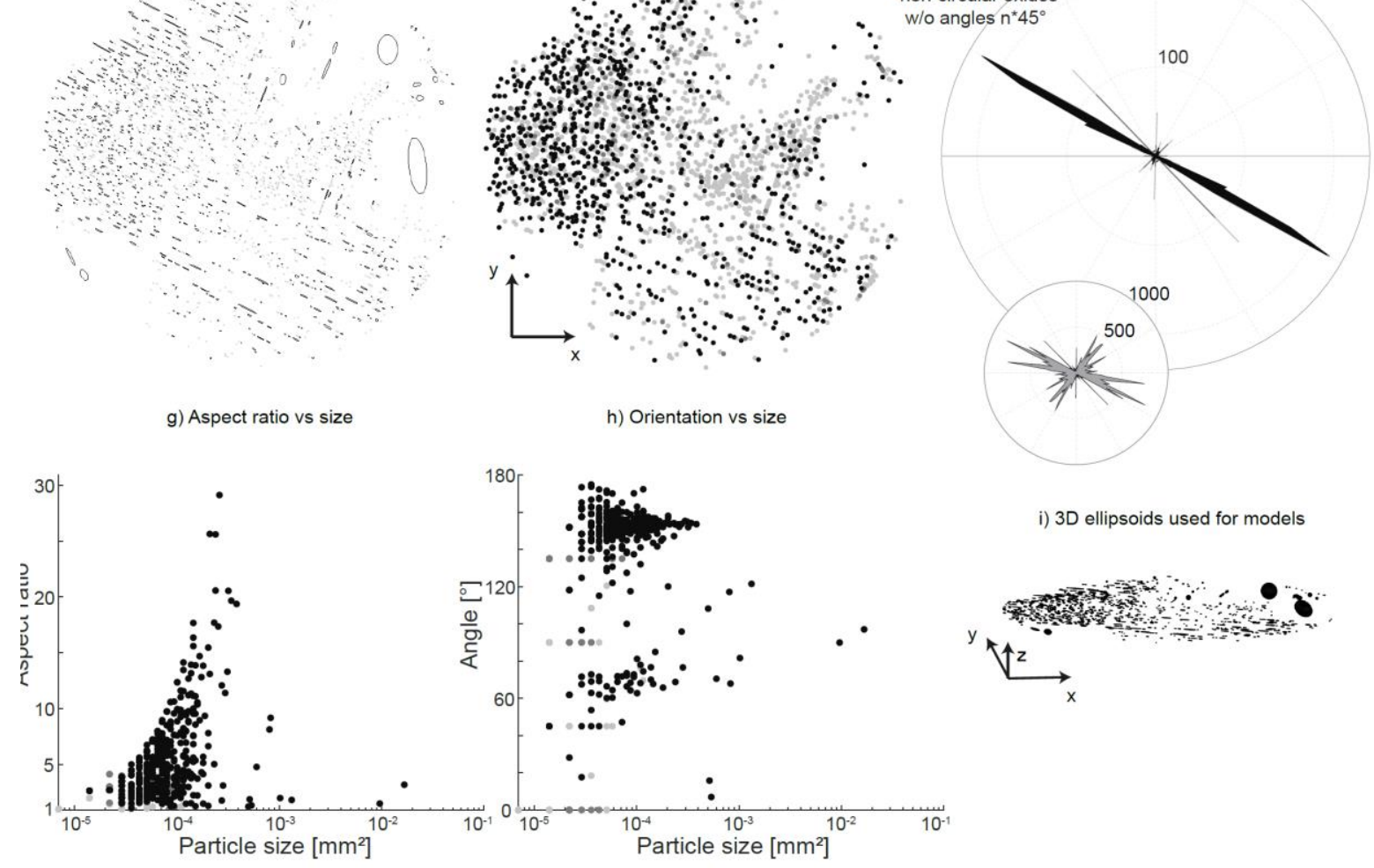

k) Modeled anisotropy directions

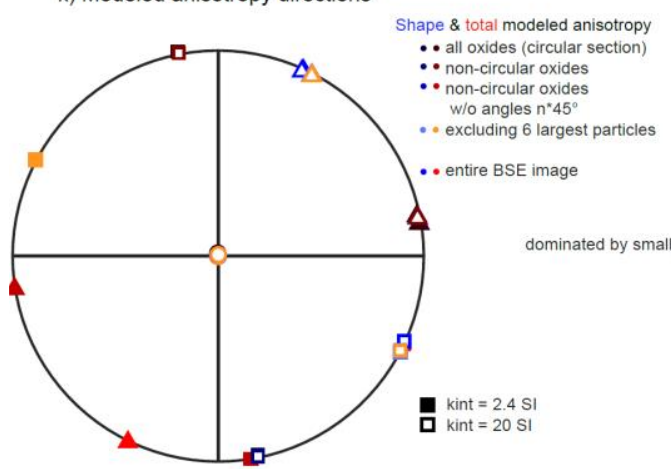

I) Modeled and measured anisotropy in image (xy) plane

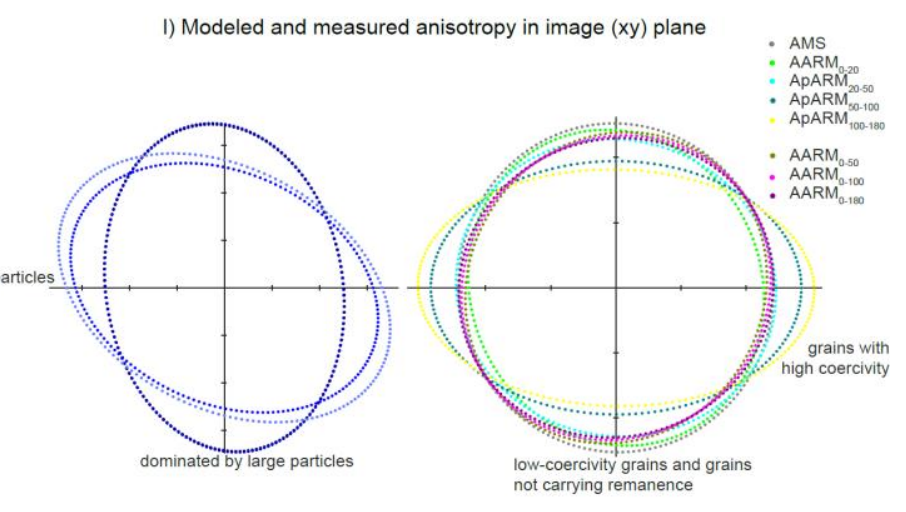

Figure 7: BSE images, isolated oxides, best-fit ellipses, and modeled and measured in-plane anisotropy for oxides in a pyroxene-bearing oxide gabbro. 


\subsection{Future applications and developments}

The FinIrrSDA code can in principle calculate the magnetic anisotropy of magnetite particles or ferrofluid-impregnated pores for (1) known pore and grain distributions in synthetic samples, (2) 3D pore or grain distributions extracted from XRCT or other structural and tomographic data, and (3) 2D pore or grain distributions obtained from image analysis. The latter is subject to certain assumptions, because demagnetization tensors need to be defined in 3D. In particular, FinIrrSDA is an improvement to previous distribution anisotropy models, in that it is not limited to regular arrangements of equal particles with the same orientation.

Nevertheless, some challenges and open questions remain. Similar to previous models, FinIrrSDA produces unrealistic results when the spacing is small with respect to the particle size. This is likely related to the assumptions of purely dipolar interaction, and homogeneous interaction field throughout the particle. Another challenge is that the model is limited to ellipsoidal or cylindrical particle shapes, because the demagnetization tensors are not defined for more complicated shapes. Particularly for networks of ferrofluid-filled pores, it needs to be defined how to describe and quantify self-demagnetization and interactions in a large connected network of highly magnetic material. More work is needed to address these issues.

All particles in the models presented here have equal intrinsic susceptibility. This is a fair assumption for ferrofluid-filled pores, as long as the fluid displays homogeneous properties throughout the pore space. Oxide particles will also have similar properties throughout the rock as long as their mineralogy, chemical composition and domain state are constant throughout the rock. If several types of oxides with different magnetic properties are present in a sample, the code can be adapted so that the magnetic properties of each particle can be defined separately. A larger change to the code will be necessary to allow for inverse fabrics of SD grains, and to describe the anisotropy in PSD grains. This will be especially relevant after expected advances in imaging/tomography techniques that also allow characterization of these small particles in standard-size paleomagnetic samples.

Currently, FinIrrSDA is limited by computation speed, in particular when calculating the distribution anisotropy for a large number of particles. This is because the mutual magnetostatic interactions for all particles on each other are calculated, and computation time increases approximately as a function of $n^{2}$, where $n$ is the number of particles, especially for large numbers of particles (Figure 8). Additional time is needed when several iterations are necessary to compute effective fields experience by each particle. This makes FinIrrSDA particularly useful for samples with low numbers of particles, e.g. synthetic samples. Because the secondary fields created by each particle are treated as independent of each other, future code developments could employ parallelization to reduce computation time for large assemblies. 


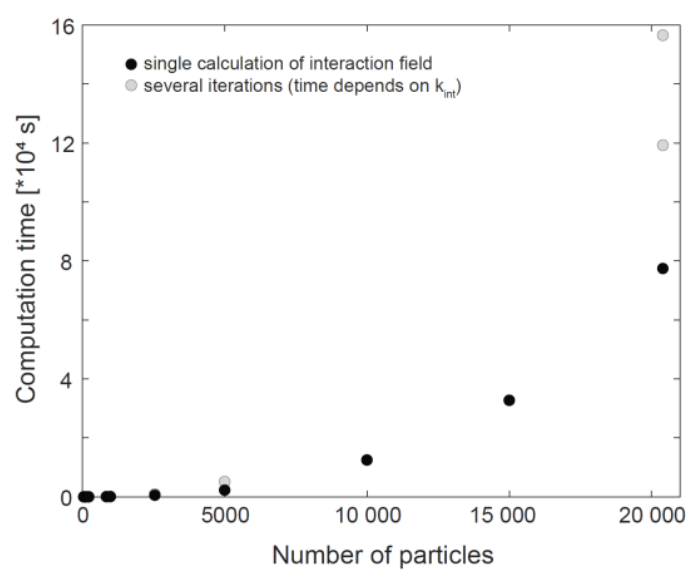

Figure 8: Computation time as a function of the number of particles in the model.

\section{Discussion and Conclusions}

Shape and distribution anisotropy together define the magnetic pore fabric of samples impregnated with ferrofluid, or the magnetic fabric of strongly magnetic particles in rock samples. A large number of factors influence the relative importance of shape and distribution anisotropy, including the particle shape, arrangement, and their magnetic properties (Cañón-Tapia, 2001). Experimental studies on synthetic samples have shown that non-random distributions of isotropic particles display anisotropy (Hargraves et al., 1991), and that both the aspect ratio of one grain (shape anisotropy) as well as the configuration of two grains (distribution anisotropy) affect the magnetic anisotropy of magnetite, where the latter was considered more important (Grégoire et al., 1995). Several modelbased studies calculated distribution anisotropy of regular arrangements of equally shaped and sized particles in infinite lines or planes (Biedermann, 2019; Cañón-Tapia, 1996, 2001; Stephenson, 1994). However, particles and pores in natural rocks have irregular shapes and sizes, and are arranged in complex patterns. Therefore, a new model is presented here, that can predict the shape anisotropy, or the combined shape and distribution anisotropy of particle assemblages more closely resembling those found in natural samples. FinIrrSDA is the first full 3D model able to compute both shape and distribution anisotropy for any particle distribution, as long as individual particles can be approximated by ellipsoids or cylinders. The input data for the model consist of a table of particle properties, and the model is thus universally applicable to a wide variety of structural data and studies.

The results presented here illustrate that distribution anisotropy depends on the number of particles, in particular when their number is small. Hence, FinIrrSDA gives more realistic results than existing distribution anisotropy models, especially in synthetic samples with a low number of particles. This effect is nicely illustrated by the magnetic pore fabrics in the synthetic samples measured by Jones et al. (2006), whose results are better matched by FinlrrSDA than by previous models based on nearest-neighbor interactions of infinite lines or planes (Biedermann, 2019).

The model presented here suggests a stronger effect of distribution anisotropy for assemblages with more than 10 particles than predicted by previous models (Biedermann, 2019; Cañón-Tapia, 1996, 2001; Stephenson, 1994). This is because FinIrrSDA takes into account more interactions, also with particles at larger distances. At the same time, because more interactions need to be calculated, FinIrrSDA is slower than the previous models. Hence, there is a trade-off between accuracy or applicability to natural samples and computation costs. Whether or not the added accuracy justifies the additional computational cost will need to be determined based on the application. 
Although FinIrrSDA provides more accurate results than previously possible, some issues remain unresolved: (1) magnetostatic interactions are calculated in the dipolar approximation, leading to artefacts if the ratio of interparticle distance to particle size is small, (2) the calculation of shape anisotropy is limited to ellipsoidal or cylindrical pores for which sufficiently accurate expressions for demagnetization factors exist. Another major limitation FinIrrSDA has in common with existing distribution anisotropy models is that the field inside each particle is approximated by the field at the particle center, which leads to inaccurate estimates of the secondary field especially for large particles at small spacing, i.e., when the contribution of distribution anisotropy is strongest. More work will be needed before magnetic anisotropy of complex shapes or entire pore networks can be modeled.

Despite its limitations, FinIrrSDA allows to predict the magnetic shape and distribution anisotropy of irregular assemblies of magnetic grains or ferrofluid-impregnated pores based on a table with the particle positions, dimensions and orientations. With these characteristics, FinIrrSDA fills a major gap between previous distribution anisotropy models and natural samples, and allows to predict the relative importance of shape and distribution anisotropy for real rocks. Thus, it facilitates our understanding of the interplay between shape and distribution anisotropy in rocks, and will help solve the controversy on the importance of each, and facilitate structural, geodynamic and fluid flow interpretations from magnetic fabrics in future studies.

\section{Acknowledgments}

Anette von der Handt, University of Minnesota, is thanked for help with the BSE imaging. An informal review by Mike Jackson is gratefully acknowledged. Comments and suggestions by Edgardo Cañón-Tapia, an anonymous reviewer, and associate editor Aleksey Smirnov helped to improve the manuscript. This study was financed by the Swiss National Science Foundation, SNSF, project 176917.

\section{Data availability}

The Matlab code is available on Zenodo, DOI: 10.5281/zenodo.4040785.

\section{References}

Baker, D. R., Mancini, L., Polacci, M., Higgins, M. D., Gualda, G. A. R., Hill, R. J., \& Rivers, M. L. (2012). An introduction to the application of X-ray microtomography to the three-dimensional study of igneous rocks. Lithos, 148, 262-276.

Balsley, J. R., \& Buddington, A. F. (1960). Magnetic susceptibility anisotropy and fabric of some Adirondack granites and orthogneisses. American Journal of Science, 258-A, 6-20.

Biedermann, A. R. (2018). Magnetic Anisotropy in Single Crystals: A Review. Geosciences, 8(8).

Biedermann, A. R. (2019). Magnetic pore fabrics: the role of shape and distribution anisotropy in defining the magnetic anisotropy of ferrofluid-impregnated samples. Geochemistry, Geophysics, Geosystems, 20(12), 5650-5666.

Biedermann, A. R., Jackson, M., Bilardello, D., \& Feinberg, J. M. (2020). Anisotropy of full and partial anhysteretic remanence across different rock types: 2 . Coercivity-dependence of remanence anisotropy. Tectonics, 39.

Biedermann, A. R., Jackson, M., Stillinger, M. D., Bilardello, D., \& Feinberg, J. M. (2020). Anisotropy of full and partial anhysteretic remanence across different rock types: 1 . Are partial anhysteretic remanence anisotropy tensors additive? Tectonics, 39.

Biedermann, A. R., Kunze, K., \& Hirt, A. M. (2018). Interpreting magnetic fabrics in amphibolebearing rocks. Tectonophysics, 722, 566-576. 
Biedermann, A. R., Pettke, T., Bender Koch, C., \& Hirt, A. M. (2015). Magnetic anisotropy in clinopyroxene and orthopyroxene single crystals. Journal of Geophysical Research-Solid Earth, 120(3), 1431-1451. Article.

Borradaile, G. (1987). Anisotropy of magnetic susceptibility: rock composition versus strain. Tectonophysics, 138, 327-329.

Borradaile, G. J., \& Henry, B. (1997). Tectonic applications of magnetic susceptibility and its anisotropy. Earth-Science Reviews, 42, 49-93.

Borradaile, G. J., \& Jackson, M. (2004). Anisotropy of magnetic susceptibility (AMS): magnetic petrofabrics of deformed rocks. In F. Martin-Hernandez, C. M. Lüneburg, C. Aubourg, \& M. Jackson (Eds.), Magnetic Fabric: Methods and Applications (Vol. 238, pp. 299-360). London, UK: Geological Society Special Publications.

Borradaile, G. J., \& Jackson, M. (2010). Structural geology, petrofabrics and magnetic fabrics (AMS, AARM, AIRM). Journal of Structural Geology, 32(10), 1519-1551.

Bown, M. G., \& Gay, P. (1959). The identification of oriented inclusions in pyroxene crystals. The American Mineralogist, 44, 592-602.

Cañón-Tapia, E. (1996). Single-grain versus distribution anisotropy: a simple three-dimensional model. Physics of the Earth and Planetary Interiors, 94, 149-158.

Cañón-Tapia, E. (2001). Factors affecting the relative importance of shape and distribution anisotropy in rocks: theory and experiments. Tectonophysics, 340, 117-131.

Clark, D. A. (1997). Magnetic petrophysics and magnetic petrology: aids to geological interpretation of magnetic surveys. Journal of Australian Geology and Geophysics, 17, 83-103.

Clark, D. A. (2014). Methods for determining remanent and total magnetizations of magnetic sources - a review. Exploration Geophysics, 45, 271-304.

Clark, D. A., \& Emerson, D. W. (1999). Self-Demagnetisation. Preview, 79, 22-25.

Crameri, F. (2018). Geodynamic diagnostics, scientific visualisation and StagLab 3.0. Geoscientific Model Development, 11(6), 2541-2562.

Dunlop, D. J., \& Özdemir, O. (1997). Rock Magnetism. Fundamentals and Frontiers. Cambridge, UK: Cambridge University Press.

Feinberg, J. M., Wenk, H.-R., Renne, P. R., \& Scott, G. R. (2004). Epitaxial relationships of clinopyroxene-hosted magnetite determined using electron backscatter diffraction (EBSD) technique. American Mineralogist, 89, 462-466.

Fleet, M. E., Bilcox, G. A., \& Barnett, R. L. (1980). Oriented magnetite inclusions in pyroxenes from the Grenville Province. Canadian Mineralogist, 18, 89-99.

Grégoire, V., Darrozes, P., Gaillot, P., \& Nédélec, A. (1998). Magnetite grain shape fabric and distribution anisotropy vs rock magnetic fabric: a three-dimensional case study. Journal of Structural Geology, 20(7), 937-944.

Grégoire, V., De Saint-Blanquat, M., Nédélec, A., \& Bouchez, J.-L. (1995). Shape anisotropy versus magnetic interactions of magnetite grains: experiments and application to AMS in granitic rocks. Geophysical Research Letters, 22(20), 2765-2768.

Hailwood, E. A., Bowen, D., Ding, F., Corbett, P. W. M., \& Whattler, P. (1999). Characterizing pore fabrics in sediments by anisotropy of magnetic susceptibility analyses. In D. H. Tarling \& P. Turner (Eds.), Paleomagnetism and Diagenesis in Sediments (Vol. 151, pp. 125-126): Geological Society, London, Special Publications.

Hargraves, R. B., Johnson, D., \& Chan, C. Y. (1991). Distribution anisotropy: the cause of AMS in igneous rocks? Geophysical Research Letters, 18(12), 2193-2196.

Hirt, A. M., \& Biedermann, A. R. (2019). Preferred orientation of ferromagnetic phases in rockforming minerals: Insights from magnetic anisotropy of single crystals. Canadian Journal of Earth Sciences.

Hirt, A. M., Lowrie, W., Clendenen, W. S., \& Kligfield, R. (1988). The correlation of magnetic anisotropy with strain in the Chelmsford Formation of the Sudbury Basin, Ontario. Tectonophysics, 145, 177-189. 
Housen, B. A., Richter, C., \& van der Pluijm, B. A. (1993). Composite magnetic anisotropy fabrics: experiments, numerical models, and implications for the quantification of rock fabrics. Tectonophysics, 220, 1-12.

Hrouda, F. (1982). Magnetic anisotropy of rocks and its application in geology and geophysics. Geophysical Surveys, 5, 37-82.

Hrouda, F., Hanak, J., \& Terzijski, I. (2000). The magnetic and pore fabrics of extruded and pressed ceramic models. Geophysical Journal International, 142, 941-947.

Jackson, M., \& Tauxe, L. (1991). Anisotropy of magnetic susceptibility and remanence: Developments in the characterization of tectonic, sedimentary, and igneous fabric. Reviews of Geophysics, 29, 371-376.

Jackson, M. J. (1991). Anisotropy of magnetic remanence: A brief review of mineralogical sources, physical origins, and geological applications, and comparison with susceptibility anisotropy. Pure and applied geophysics, 136, 1-28.

Jelinek, V. (1977). The statistical theory of measuring anisotropy of magnetic susceptibility of rocks and its application.

Jelinek, V. (1996). Measuring anisotropy of magnetic susceptibility on a slowly spinning specimen basic theory. AGICO Print No. 10.

Jones, S., Benson, P., \& Meredith, P. (2006). Pore fabric anisotropy: testing the equivalent pore concept using magnetic measurements on synthetic voids of known geometry. Geophysical Journal International, 166, 485-492.

Ketcham, R. A. (2005). Three-dimensional grain fabric measurements using high-resolution X-ray computed tomography. Journal of Structural Geology, 27(7), 1217-1228.

Kligfield, R., Lowrie, W., \& Dalziel, W. D. (1977). Magnetic susceptibility anisotropy as a strain indicator in the Sudbury Basin, Ontario. Tectonophysics, 40, 287-308.

Kligfield, R., Owens, W. H., \& Lowrie, W. (1981). Magnetic susceptibility anisotropy, strain, and progressive deformation in Permian sediments from the Maritime Alps (France). Earth and Planetary Science Letters, 55, 181-189.

Landis, E. N., \& Keane, D. T. (2010). X-ray microtomography. Materials Characterization, 61(12), 1305-1316.

Launeau, P., \& Cruden, A. R. (1998). Magmatic fabric acquisition mechanisms in a syenite: Results of a combined anisotropy of magnetic susceptibility and image analysis study. Journal of Geophysical Research: Solid Earth, 103(B3), 5067-5089.

Lowrie, W. (1997). Fundamentals of Geophysics. Cambridge, UK: Cambridge University Press.

Martín-Hernández, F., \& Ferré, E. C. (2007). Separation of paramagnetic and ferrimagnetic anisotropies: A review. Journal of Geophysical Research-Solid Earth, 112(B3).

Nabawy, B. S., Rochette, P., \& Géraud, Y. (2009). Petrophysical and magnetic pore network anisotropy of some cretaceous sandstone from Tushka Basin, Egypt. Geophysical Journal International, 177, 43-61.

Osborn, J. A. (1945). Demagnetizing factors of the general ellipsoid. Physical Review, 67(11-12), 351357.

Parés, J., Miguens, L., \& Saiz, C. (2016). Characterizing pore fabric in sandstones with magnetic anisotropy methods: initial results. Journal of Petroleum Science and Engineering, 143, 113120.

Pfleiderer, S., \& Halls, H. C. (1990). Magnetic susceptibility anisotropy of rocks saturated with ferrofluid: a new method to study pore fabric? Physics of the Earth and Planetary Interiors, 65, 158-164.

Pfleiderer, S., \& Halls, H. C. (1993). Magnetic pore fabric analysis: Verification through image autocorrelation. Journal of Geophysical Research, 98(B3), 4311-4316. 
Pfleiderer, S., \& Halls, H. C. (1994). Magnetic pore fabric analysis: a rapid method for estimating permeability anisotropy. Geophysical Journal International, 116, 39-45.

Pugnetti, M., Zhou, Y., \& Biedermann, A. R. (2020). Testing the efficienty of ferrofluid impregnation in porous media: Recommendations for future magnetic pore fabric studies. Paper presented at the EGU Sharing Geoscience Online.

Rochette, P., Aubourg, C., \& Perrin, M. (1999). Is this magnetic fabric normal? A review and case studies in volcanic formations. Tectonophysics, 307, 219-234.

Rochette, P., Jackson, M., \& Aubourg, C. (1992). Rock magnetism and the interpretation of anisotropy of magnetic susceptibility. Reviews of Geophysics, 30(3), 209-226.

Sato, M., \& Ishii, Y. (1989). Simple and approximate expressions of demagnetizing factors of uniformly magnetized rectangular rod and cylinder. Journal of Applied Physics, 66(2), 983985.

Schneider, C. A., Rasband, W. S., \& Eliceiri, K. W. (2012). NIH image to ImageJ: 25 years of image analysis. Nature Methods, 9(7), 671-675.

Stacey, F. D., \& Banerjee, S. K. (1974). The Physical Principles of Rock Magnetism (Vol. 5). Amsterdam, Netherlands: Elsevier.

Stephenson, A. (1994). Distribution anisotropy: two simple models for magnetic lineation and foliation. Physics of the Earth and Planetary Interiors, 82, 49-53.

Stoner, E. C. (1945). The demagnetizing factors for ellipsoids. The London, Edinburgh, and Dublin Philosophical Magazine and Journal of Science: Series 7, 36(263), 803-821.

Tarling, D. H., \& Hrouda, F. (1993). The magnetic anisotropy of rocks. London, UK: Chapman and Hall. Tauxe, L., Banerjee, S. K., Butler, R. F., \& van der Voo, R. (2018). Essentials of Paleomagnetism (5th Web Edition). https://earthref.org/MagIC/books/Tauxe/Essentials/.

Thompson, R., \& Oldfield, F. (1986). Environmental Magnetism. London, UK: Allen \& Unwin. 